\title{
A Convex Variational Model for Restoring SAR Images Corrupted by Multiplicative Noise
}

\author{
Hanmei Yang, ${ }^{1,2}$ Jiachang $\mathrm{Li}^{2}{ }^{2}$ Lixin Shen, ${ }^{3}$ and Jian $\mathrm{Lu} \mathbb{1}^{2,4}$ \\ ${ }^{1}$ School of Software Engineering, Tongii University, Shanghai 201804, China \\ ${ }^{2}$ Shenzhen Key Laboratory of Advanced Machine Learning and Applications, College of Mathematics and Statistics, \\ Shenzhen University, Shenzhen 518060, China \\ ${ }^{3}$ Department of Mathematics, Syracuse University, Syracuse, NY 13244, USA \\ ${ }^{4}$ Guangdong Key Laboratory of Intelligent Information Processing, Shenzhen University, Shenzhen 518060, China
}

Correspondence should be addressed to Jian Lu; jianlu1979@163.com

Received 29 August 2019; Revised 12 February 2020; Accepted 17 April 2020; Published 10 June 2020

Academic Editor: Daniel Zaldivar

Copyright $(2020$ Hanmei Yang et al. This is an open access article distributed under the Creative Commons Attribution License, which permits unrestricted use, distribution, and reproduction in any medium, provided the original work is properly cited.

\begin{abstract}
This paper studies a new convex variational model for denoising and deblurring images with multiplicative noise. Considering the statistical property of the multiplicative noise following Nakagami distribution, the denoising model consists of a data fidelity term, a quadratic penalty term, and a total variation regularization term. Here, the quadratic penalty term is mainly designed to guarantee the model to be strictly convex under a mild condition. Furthermore, the model is extended for the simultaneous denoising and deblurring case by introducing a blurring operator. We also study some mathematical properties of the proposed model. In addition, the model is solved by applying the primal-dual algorithm. The experimental results show that the proposed method is promising in restoring (blurred) images with multiplicative noise.
\end{abstract}

\section{Introduction}

Image restoration problems [1-9] have attracted a great amount of attention in real applications. Noise in images is often roughly grouped into additive noise and multiplicative noise. In this paper, we mainly focus on the multiplicative noise removal problem [10], which can be formulated as

$$
f=(K u) v \text {, }
$$

where $f: \Omega \longrightarrow \mathbb{R}^{+}$is the observed image $\left(\Omega \subset \mathbb{R}^{2}\right.$ is a connected bounded open subset with compact Lipschitz boundary), $v \in L^{2}(\Omega)$ denotes multiplicative noise with mean 1 , and $K \in \mathscr{L}\left(L^{2}(\Omega)\right)$ is a known linear and continuous blurring operator. The goal is to find the unknown true image $u$ from the degraded image $f$ as well as possible.

Multiplicative noise, also known as speckle [11-15], often occurs in active imaging systems, such as synthetic aperture radar (SAR), laser images, and ultrasound imaging. In this paper, the characteristics of speckle which follow a Nakagami distribution in SAR images are considered. In
SAR imaging system which are widely used in military reconnaissance, marine monitoring, and other fields, a radar sends a coherent wave. Then, it is reflected on the ground and "recorded" by the radar sensor to capture SAR images. The fading of radar echo signal often causes that the SAR images are degraded by speckle, which limits their interpretation and processing.

Over the past decades, there is a great deal of research that has been done on solving the speckled image problem [16-19]. Early filtering techniques are exploited under the minimum mean square error (MMSE) criterion in the spatial domain [20]. And the bilateral filtering [21], the meshless method [22], the low-rank method [23], and the wavelet-based [24, 25] and nonlocal-based [26-28] approaches have also been explored for despeckling. Particularly, in some statistics-based methods, the observed image intensity is considered as a random variable following a negative exponential law, and then the intensity of the $L$-look image which is obtained as the average of $L$ different independent images follows a gamma law with mean equal 
to one. And thus, the image to be recovered is assumed to be degraded by multiplicative gamma noise. Based on this assumption, a series of variational models, including the ZWM model [29], the AA model, and its variants and convexity improvements (e.g., the exponent-based models [30-33], the $m \mathrm{~V}$ model [34], the TwL- $m \mathrm{~V}$ model [35], and DZ model [36]), are proposed, where the regularization terms generally play a key role to preserve the image structure such as edge detail. On the contrary, in some other statistics-based methods, the image amplitude which is the square root of the reflectivity (intensity) image is taken into consideration. And the pixel amplitudes are modeled as independent and identically distributed according to the Nakagami distribution. Related work includes the probabilistic patch-based (PPB) method [27], the SAR blockmatching 3D (SAR-BM3D) method [28], DD-SRAD method [37], a variation-based model by using fields of experts (FoEs) [38] proposed by Chen et al. [11], and the references cited therein.

Based on the works $[11,36,39]$, we investigate a new convex variational model in this paper, where the image amplitude is considered, and the fidelity term is deduced from the probability density function of the Nakagami distribution and a quadratic penalty term is derived from the statistical property of Nakagami distribution, which guarantees the proposed model to be strictly convex under a mild condition. The convex model guarantees the uniqueness of the solution. Under the framework of continuous functional, we study the existence and uniqueness of the proposed model to the image denoising and deblurring problem. Furthermore, we employ the primal-dual method $[36,40]$ to solve the proposed model. Finally, we arrange some numerical comparisons on simulated images and real SAR images to illustrate the efficiency of the model.

\section{Methods}

To recover $u$ for the degraded image $f$ in (1), based on the statistical properties of Nakagami noise, in Section 2.1, we first propose a new variational model (6) for image denoising (i.e., $K=I$ ). We analyze the existence and uniqueness of the solution of the new model and discuss the properties of the solution of the model. Then, we extend the denoising model (6) to simultaneous deblurring and denoising case and also show the properties of the solution of the model in Section 2.2. And on these bases, we introduce a primal-dual algorithm to solve the proposed model in Section 2.3. By adopting the similar ideas in $[36,39]$, we can get Propositions $1,2-5$, and Theorem 1. And the proof of Propositions 1,2 , and 4 is given in the Appendices $\mathrm{A}, \mathrm{C}$, and $\mathrm{D}$, respectively; and the proof of Theorem 1 can be found in Appendix B.

2.1. A Convex Variational Denoising Model. We now introduce a new variational model for image denoising, based on the statistical properties of Nakagami noise. For the multiplicative model $f=u v$, we assume that $v$ follows a
Nakagami distribution, the square root of the reflectivity $[11,13]$ :

$$
p(f \mid u)=\frac{2 L^{L}}{\Gamma(L) u^{2 L}} f^{2 L-1} e^{-\left(L f^{2} / u^{2}\right)},
$$

where $L$ is the number of looks of the image (i.e., number of independent values averaged) and $\Gamma(\cdot)$ is the classical Gamma function.

Based on (2), by using the MAP estimator and combining the resulting data term with the total variation (TV) prior model, we obtain the following AA-like variational model:

$$
\inf _{u \in \bar{S}(\Omega)} \int_{\Omega}\left(2 \log u+\frac{f^{2}}{u^{2}}\right) \mathrm{d} x+\lambda \int_{\Omega}|D u|,
$$

where $\lambda>0$ and $\bar{S}(\Omega):=\{u \in B V(\Omega): u \geq 0\}$. And $\int_{\Omega}|D u|$ is the TV of image $u$.

Let $z=(1 / v)$ and $z$ denote the instance of random variable $Z$. It is not hard to get that its PDF is

$$
p_{Z}(z)=\frac{2 L^{L}}{\Gamma(L)} z^{-2 L-1} e^{-L / z^{2}}, \quad \forall z \geq 0 .
$$

A direct calculation shows that

$$
\begin{aligned}
\mathbb{E}(Z) & =\frac{\sqrt{L} \Gamma(L-(1 / 2))}{\Gamma(L)}>0, \\
\lim _{L \longrightarrow+\infty} \mathbb{E}\left((Z-1)^{2}\right) & =0 .
\end{aligned}
$$

Based on this observation, we therefore introduce a quadratic penalty term $\int_{\Omega}((u / f)-\beta 1)^{2} \mathrm{~d} x$ into the AA-like model (3), yielding the following optimization problem:

$$
\begin{aligned}
\inf _{u \in \bar{S}(\Omega)} E(u):= & \int_{\Omega}\left(2 \log u+\frac{f^{2}}{u^{2}}\right) \mathrm{d} x+\alpha \int_{\Omega}\left(\frac{u}{f}-\beta 1\right)^{2} \mathrm{~d} x \\
& +\lambda \int_{\Omega}|D u|
\end{aligned}
$$

where $\alpha>0, \lambda>0$, and $\beta \geq 1$ vary with the level of the noise. We now show that $E(u)$ in (6) is convex if the parameter $\alpha$ satisfies certain condition.

Proposition 1. If $\alpha$ is chosen satisfying

$$
\alpha \geq \frac{1}{12},
$$

then the model (6) is strictly convex.

Next, we study the existence and uniqueness of the solution to (6) and the minimum-maximum principle. To this end, we make the following notations. Given a function $f \in L^{\infty}(\Omega)$, we denote by $\sup _{\Omega} f$ (resp., $\inf _{\Omega} f$ ) the supremum (resp., infimum) of $f$, i.e., $\sup _{\Omega} f=$ $\inf \{C \in \mathbb{R} ; f(x) \leq C$ a.e. $\} \quad$ (resp., $\inf _{\Omega} f=\sup \{C \in \mathbb{R} ; f(x)$ $\geq C$ a.e. $\}$ ). 
Theorem 1. If $f \in L^{\infty}(\Omega)$ and $\inf _{\Omega} f>0$, then the problem (6) has a solution $u^{*} \in B V(\Omega)$ such that

$$
0<\inf _{\Omega} f \leq u^{*} \leq \beta \sup _{\Omega} f .
$$

Moreover, the solution of (6) is unique when $\alpha$ is chosen to satisfy (7).

We now introduce a comparison principle that characterizes the relationship of solutions of problem (6) with different $f$ values.

Proposition 2. Suppose $f_{1}$ and $f_{2}$ are in $L^{\infty}(\Omega)$ with $\inf _{\Omega} f_{1}>0$ and $\inf _{\Omega} f_{2}>0$. Let $a_{1}=\inf _{\Omega} f_{1}, a_{2}=\inf _{\Omega} f_{2}$, $b_{1}=\sup _{\Omega} f_{1}$, and $b_{2}=\sup _{\Omega} f_{2}$. Assume that $f_{1}<f_{2}$. We denote by $u_{1}^{*}$ (resp., $u_{2}^{*}$ ) a solution of (6) for $f=f_{1}$ (resp., $\left.f=f_{2}\right)$. Then, we have $u_{1}^{*} \leq u_{2}^{*}$ a.e. in $\Omega$ if $\alpha$ and $\beta$ satisfy $(\alpha \beta / 1+\alpha)<\left(a_{1}^{2} a_{2}^{2} / b_{1}^{2} b_{2}^{2}\right)$

2.2. A Simultaneous Deblurring and Denoising Model. Given a nonnegative, linear, and continuous blurring operator $K \in \mathscr{L}\left(L^{2}(\Omega)\right)$, the degraded image can be expressed as $f=(K u) v$. Here, $f$ is the observed image, $u$ is the image to be recovered, and $v$ is the noise. Similar to (6), the deblurring and denoising model can be formulated as

$$
\begin{aligned}
\inf _{u \in \bar{S}(\Omega)} E_{K}(u):= & \int_{\Omega}\left(2 \log (K u)+\frac{f^{2}}{(K u)^{2}}\right) \mathrm{d} x \\
& +\alpha \int_{\Omega}\left(\frac{K u}{f}-\beta 1\right)^{2} \mathrm{~d} x+\lambda \int_{\Omega}|D u| .
\end{aligned}
$$

Note that (6) is a special case of (9) with $K$ being the identity operator.

We can show that the above model (9) is convex if $\alpha$ satisfies some certain conditions.

Proposition 3. If $\alpha$ is chosen satisfying

$$
\alpha \geq \frac{1}{12}
$$

then the model (9) is convex.

In view of Proposition convexdesp and the fact that $K$ is linear, it is not hard to show that the conclusion of Proposition 3holds.

We next show that $K u^{*}$ is positive; i.e., $\left\{x \in \Omega:\left(K u^{*}\right)(x)=0\right\}$ has a zero Lebesgue measure when $\varepsilon$ approaches to zero. And furthermore, we can obtain that $K u^{*}$ is strictly positive in the discrete situation.

Proposition 4. Suppose $f \in L^{\infty}(\Omega)$ and $\inf _{\Omega} f>0$. Let $K \in \mathscr{L}\left(L^{2}(\Omega)\right)$ be a linear and continuous blurring operator. Assume that $u^{*}$ is the solution of (9). Then, there exists a constant $C$ such that, for any $0<\varepsilon<1$,

$$
\begin{aligned}
\left|\left\{x \in \Omega:\left(K u^{*}\right)(x) \leq \varepsilon f(x)\right\}\right| \leq & \frac{\varepsilon^{2}}{1+2 \varepsilon^{2} \log \varepsilon} \\
& \cdot\left(C-2 \int_{\Omega} \log f \mathrm{~d} x\right) .
\end{aligned}
$$

The following Proposition 5 implies that, in general, model (9) cannot automatically preserve the mean of the original image.

Proposition 5. Let $u^{*}$ be a solution of (7). Suppose that $K 1=$ 1 and $\inf _{\Omega} K u^{*}>0$. The following statements hold:

(i) $\int_{\Omega}\left[\left(f^{2} /\left(K u^{*}\right)^{3}\right)-\alpha\left(\left(K u^{*} / f^{2}\right)-(\beta 1 / f)\right)\right] d x=$
$\int_{\Omega}\left(1 / K u^{*}\right) d x$.

(ii) If there exists a solution of (10) for $\alpha=0$, then

$$
\int_{\Omega} \frac{K u^{*}}{f^{2}} \mathrm{~d} x \geq \int_{\Omega} \frac{1}{K u^{*}} \mathrm{~d} x .
$$

We can enhance the model (9) to reduce the influence from the bias by keeping the same mean of the recovered image as $f$ by the following model:

$$
\begin{aligned}
& \inf _{\left\{u \in \bar{S}(\Omega): \mathbb{E}_{\Omega}(u)=\mathbb{E}_{\Omega}(f)\right\}} \int_{\Omega}\left(2 \log K u+\frac{f^{2}}{(K u)^{2}}\right) \mathrm{d} x \\
& +\alpha \int_{\Omega}\left(\frac{K u}{f}-\beta 1\right)^{2} \mathrm{~d} x+\lambda \int_{\Omega}|D u|,
\end{aligned}
$$

where $\mathbb{E}_{\Omega}(f)$ is the mean value of $f$. Due to the fact that $\left\{u \in \bar{S}(\Omega): \mathbb{E}_{\Omega}(u)=\mathbb{E}_{\Omega}(f)\right\}$ is closed and convex, it is easy to see that (13) has exactly one solution.

2.3. An Iterative Algorithm. For the sake of developing numerical algorithms in real-world applications, in this section, we discuss a discrete version of model (6). For simplicity, we use the same notation as the abovementioned continuous version. Here, let $K \in \mathbb{R}^{d \times d}, Q=\left\{u \in \mathbb{R}^{d}\right.$ : $u_{i} \geq 0, i=1, \ldots, d$, and $\left.\sum_{i=1}^{d} u_{i}=\sum_{i=1}^{d} f_{i}\right\}$, and the images $u, f \in \mathbb{R}^{d}$, and $\nabla \in \mathbb{R}^{2 d \times d}$ be the discrete gradient operator with $\nabla_{x}$ and $\nabla_{y}$ being, respectively, the discrete derivatives in the $x$-direction and $y$-direction. With the above notations, the discrete version of model (6) is given as follows:

$$
\min _{u \in Q} E_{K}(u):=F_{K}(u)+\lambda\|u\|_{T V}
$$

where

$$
\begin{aligned}
F_{K}(u) & :=\langle 2 \log K u, 1\rangle+\left\langle\frac{f^{2}}{(K u)^{2}}, 1\right\rangle+\alpha\left\|\frac{K u}{f}-\beta 1\right\|_{2}^{2}, \\
\|u\|_{T V} & =\sum_{i=1}^{d} \sqrt{\left(\nabla_{x} u\right)_{i}^{2}+\left(\nabla_{y} u\right)_{i}^{2}} .
\end{aligned}
$$


The minimization problem (14) can be solved by many numerical algorithms, such as the split-Bregman algorithm [41], the ADMM [42], and proximity algorithm [43]. In this section, we adopt the primal-dual algorithm to solve the problem (14) by formulating it as

$$
\max _{p \in P} \min _{u \in Q} F_{K}(u)-\lambda\langle u, \operatorname{div} p\rangle,
$$

where div is the divergence operator and $P=$ $\left\{p \in \mathbb{R}^{2 d}: \max _{i \in\{1, \ldots, d\}}\left|\left(p_{i}^{2}+p_{i+d}^{2}\right)^{1 / 2}\right| \leq 1\right\}$ with $p$ being the dual variable.

Now, similar to [36], we present the primal-dual algorithm in Algorithm 1. In order to ensure the iterates $\left\{\left(u^{n}, p^{n}\right)\right\}$ of Algorithm 1 converge to a saddle point of (14), the parameters $\mu, \rho$, and $\lambda$ should satisfy $\mu \rho \lambda^{2}<1 / 8$ $[36,44]$. And for simplification, we set $\mu=\rho=3$ in our experiments.

\section{Results and Discussion}

In this section, we conduct numerical experiments on simulated images and real SAR images to evaluate the approximation accuracy and computational efficiency of our proposed algorithm. We compare our method in the denoising case with the Lee filters [20], the speckle reducing anisotropic diffusion (SRAD) method [45], the probabilistic patch-based (PPB) method [27], and the model (13) with $\alpha=0$ using the algorithm adopted in [39] (here, we call it AA-like method), while in the deblurring and denoising case, our method is compared with the AA-like method. In order to preserve the mean of the observed image, the bias correction technique [36] is utilized for the AA-like method and our method. All the experiments are performed in MATLAB R2017b on a laptop with Intel(R) Core(TM) i7-5500U central processing unit (CPU, $2.40 \mathrm{GHz}), 8.0 \mathrm{G}$ memory, and Windows 8.1 operating system.

In our simulated test, we choose images of "Boat" and "Lena" of size $512 \times 512$, "Cameraman" of size $256 \times 256$, "Remote1" of size $460 \times 460$, and "Remote2" of size $570 \times 570$, which are shown in Figure 1 .

The quality of recovered images obtained from various despeckling algorithms is evaluated by the structural similarity index (SSIM) [46] and the peak signal-to-noise ratio (PSNR):

$$
\operatorname{PSNR}(u, \widehat{u})=10 \log _{10}\left(\frac{255^{2} d}{\|u-\widehat{u}\|_{2}}\right),
$$

where $u$ and $\widehat{u}$ denote the original image and the recovered image, respectively; and $d$ is the total number of pixels of $u$. All the methods except PPB and Lee filter are terminated by the following stopping criterion:

$$
\frac{\left\|u^{n}-u^{n+1}\right\|_{2}}{\left\|u^{n}\right\|_{2}}<\varepsilon
$$

where $\varepsilon$ is a fixed threshold. We set $\varepsilon=1.5 \times 10^{-4}$ in the denoising experiment and $\varepsilon=2 \times 10^{-5}$ in the deblurring experiment.
3.1. Denoising Experiment. The first experiment focuses on the denoising case; i.e., $K$ in model (14) is the identity operator. We first choose the test image of "Boat" and degrade it by multiplicative Nakagami noise with $L=10$. With a large value of $L$, the value of $\beta$ is 1 . We denote Algorithm 2, Algorithm 3, and Algorithm 4 by the same algorithm framework of Algorithm 1 but with the initialization replaced by $u^{0}=\bar{u}^{0}=u_{\mathrm{PPB}}, \quad u_{\text {Lee }}, u_{\mathrm{SRAD}}$, and $p^{0}=(0, \ldots, 0)^{T} \in \mathbb{R}^{2 d}$. Here, $u_{\mathrm{PPB}}, u_{\text {Lee }}$, and $u_{\mathrm{SRAD}}$ are the solutions of the PPB method [27], Lee filter [20], and SRAD method [45], respectively. The parameter settings of four algorithms are $\lambda=0.01, \alpha=2.7$, and $\beta=1$. It can be seen from Figure 2 that although Algorithms 1, 2, 3, and 4 using the same parameters converge to the same stable PSNR value in accordance with the strict convexity and the uniqueness of the solution of the model, the curve of PSNR values associated with Algorithm 2 has a higher peak than that of Algorithms 1, 3, and 4. AA-like method with the same initial guess (named the PPB-AA method) is also added for making the experiments more persuading. Based on this observation, Algorithm 2, PPB-AA, together with Algorithm 1, Lee filter, SRAD method, PPB method, and AA-like method are listed in Table 1 to make a comparison. In Table 1, the images are corrupted by multiplicative noise (Nakagami distribution with $L=10, L=7$, and $L=5$ ). The smaller the $L$ is, the noisier the images are. We tune the parameters to insure a better performance for every image. The parameter settings of proposed algorithms are shown in Table 2. For the Lee filter, the default window size of 5 is used. For the SRAD method, the parameters are set as suggested in [45]. For the PPB method, we use the codes provided by the work in [27]. And for PPB-AA and Algorithm 2, the computing time is expressed as the sum of the CPU cost of getting the initial solution $u_{\mathrm{PPB}}$ and true CPU time of Algorithm 2 and AA-like method, respectively. We observe that the PSNR and SSIM values of the restored images by Algorithm 1 are always higher than those of AA-like method, which indicates that the choices of $\alpha$ and $\beta$ have a significant impact on the quality of denoised images. Moreover, the PSNR and SSIM values of the restored images by Algorithm 2 are generally the highest among all those seven methods tested.

To visually compare the image restoration performance of above seven methods, Figures 3 and 4 report the denoising results of "Lena" and "Remotel" with $L=5$, and the certain corresponding partial enlarged denoising images of "Lena" and "Remotel" with $L=10, L=7$, and $L=5$ by these methods are shown in Figures 5-10, respectively. Firstly, we find that much noise remains in the results of Lee filter, SRAD method, and AA-like method (e.g., the hat of "Lena" shown in Figures 5-7 and roofs in "Remotel" shown in Figures 8-10). Lee filter leads to a blurred effect on restored images. Since the AA-like method is oversmoothing in the process of despeckling, details of images tend to be reduced. Conversely, more details are preserved by Algorithm 1 and SRAD method. Secondly, the PPB method in [27] can remove the multiplicative noise effectively because it is a patch-based method, which is a different framework from TV-based methods (e.g., AA-like method and Algorithm 1). The images obtained from the PPB method provide 


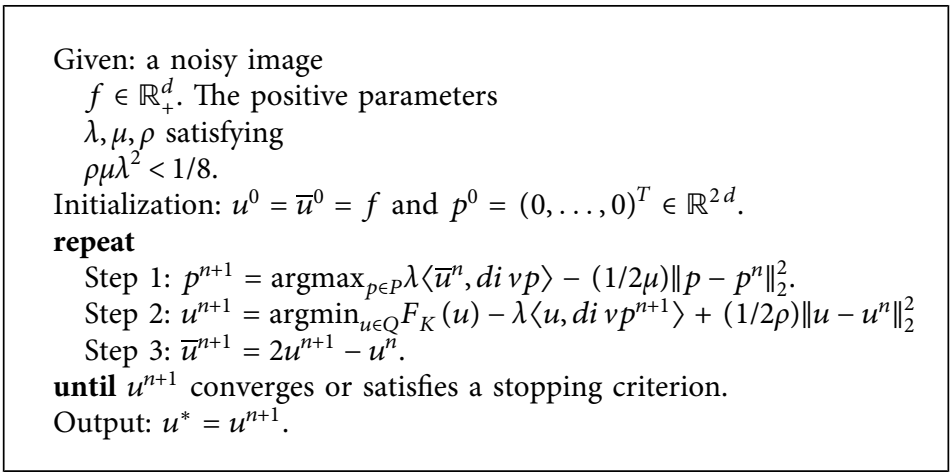

Algorithm 1: Primal-dual iterative algorithm for solving model (14).

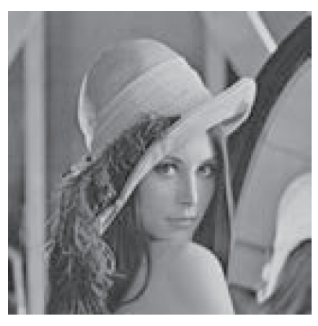

(a)

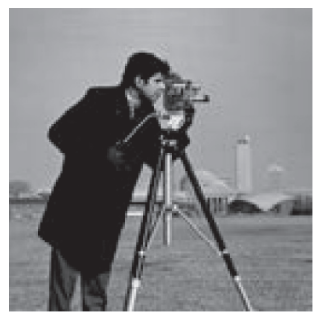

(b)

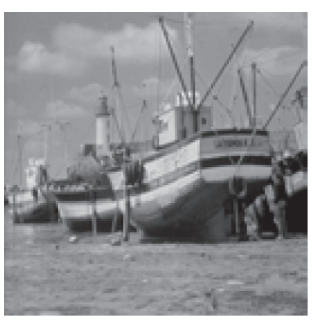

(c)

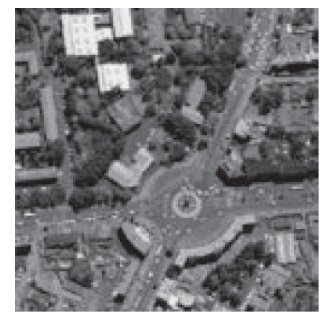

(d)

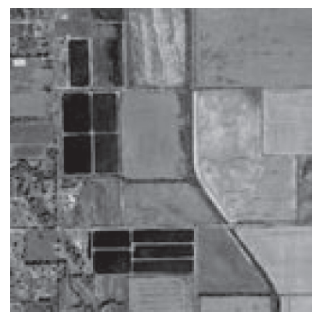

(e)

Figure 1: The five test images: (a) Lena; (b) Cameraman; (c) Boat; (d) Remote1; (e) Remote2.

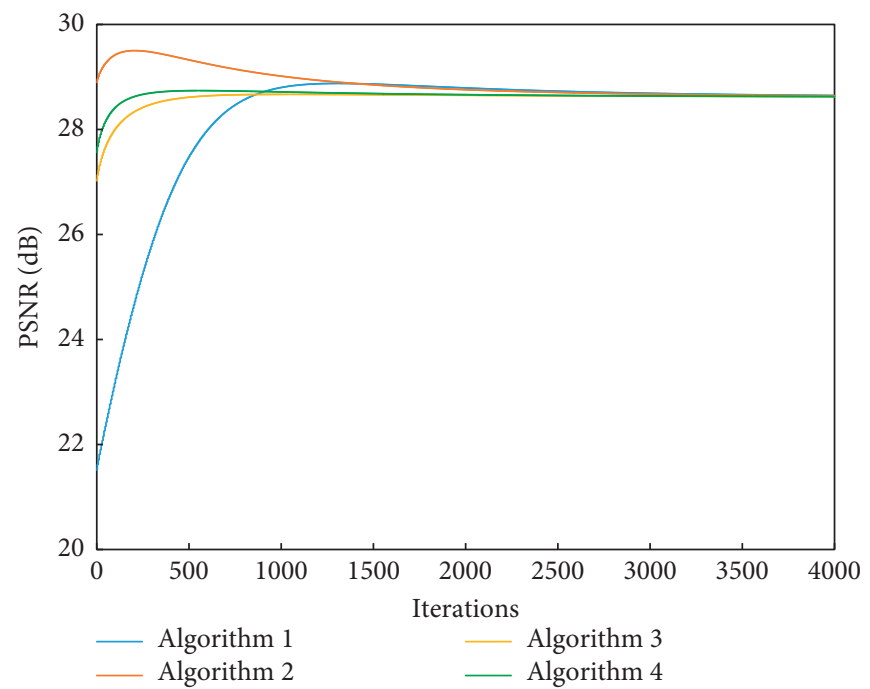

FIGURE 2: The PSNR values against the number of iterations on Boat image using Algorithm 1, Algorithm 2, Algorithm 3, and Algorithm 4.

smoother regions and better shape preservation (e.g., "Remote1" shown in Figure 4 and the backgrounds in "Lena" shown in Figure 3). However, we still notice some artifacts (e.g., the edge of the homogeneous regions in "Lena" and "Remote1"), and the PPB method seems to attenuate the image sharpness (e.g., the hat of "Lena" and trees in "Remote1") shown in Figures 5-10. Indeed, those spurious artifacts can be removed by the TV regularization in our method, but Algorithm 1 may suffer from staircasing effects (e.g., the skin of "Lena"). Finally, we observe that Algorithm 2, which combines Algorithm 1 and PPB method, can resolve the problems of PPB method and Algorithm 1 and performs better than the PPB-AA method in attenuating the unpleasant artifacts. 
TABLE 1: The comparison of different denoising methods.

\begin{tabular}{|c|c|c|c|c|c|c|c|c|c|c|}
\hline \multirow{2}{*}{ Image } & \multirow{2}{*}{ Method } & \multicolumn{3}{|c|}{$L=10$} & \multicolumn{3}{|c|}{$L=7$} & \multicolumn{3}{|c|}{$L=5$} \\
\hline & & PSNR & SSIM & Time (s) & PSNR & SSIM & Time & PSNR & SSIM & Time (s) \\
\hline \multirow{7}{*}{ Lena } & Lee & 29.52 & 0.872 & 0.22 & 28.58 & 0.847 & 0.22 & 27.75 & 0.816 & 0.19 \\
\hline & SRAD & 29.75 & 0.896 & 1.55 & 29.42 & 0.873 & 1.22 & 29.02 & 0.877 & 1.68 \\
\hline & AA-like & 30.01 & 0.882 & 50 & 29.01 & 0.864 & 58 & 28.12 & 0.844 & 71 \\
\hline & Algorithm 1 & 30.99 & 0.910 & 89 & 29.95 & 0.895 & 105 & 28.91 & 0.874 & 125 \\
\hline & PPB & 31.82 & 0.935 & 52 & 31.08 & 0.924 & 51 & 30.37 & 0.909 & 50 \\
\hline & PPB-AA & 32.05 & 0.932 & $52+6$ & 31.09 & 0.921 & $51+6$ & 30.18 & 0.908 & $50+7$ \\
\hline & Algorithm 2 & 32.28 & 0.938 & $52+23$ & 31.29 & 0.928 & $51+23$ & 30.82 & 0.922 & $50+32$ \\
\hline \multirow{7}{*}{ Boat } & Lee & 27.00 & 0.839 & 0.25 & 26.29 & 0.814 & 0.24 & 25.57 & 0.786 & 0.20 \\
\hline & SRAD & 27.54 & 0.856 & 1.11 & 27.28 & 0.842 & 1.09 & 26.84 & 0.825 & 1.17 \\
\hline & AA-like & 28.30 & 0.867 & 41 & 27.42 & 0.845 & 53 & 26.55 & 0.818 & 64 \\
\hline & Algorithm 1 & 28.90 & 0.885 & 92 & 28.02 & 0.863 & 109 & 27.11 & 0.839 & 125 \\
\hline & $\mathrm{PPB}$ & 29.01 & 0.884 & 52 & 28.24 & 0.858 & 51 & 27.53 & 0.835 & 51 \\
\hline & PPB-AA & 29.07 & 0.875 & $52+5$ & 28.19 & 0.849 & $51+5$ & 27.33 & 0.826 & $51+6$ \\
\hline & Algorithm 2 & 29.44 & 0.891 & $52+27$ & 28.49 & 0.865 & $51+29$ & 28.02 & 0.851 & $51+40$ \\
\hline \multirow{7}{*}{ Cameraman } & Lee & 27.11 & 0.757 & 0.12 & 26.07 & 0.719 & 0.14 & 25.06 & 0.686 & 0.13 \\
\hline & SRAD & 27.46 & 0.753 & 0.32 & 27.06 & 0.740 & 0.28 & 26.61 & 0.760 & 0.35 \\
\hline & AA-like & 27.91 & 0.759 & 9 & 26.85 & 0.740 & 11 & 25.93 & 0.729 & 14 \\
\hline & Algorithm 1 & 28.46 & 0.767 & 26 & 27.77 & 0.794 & 23 & 26.84 & 0.774 & 27 \\
\hline & $\mathrm{PPB}$ & 28.09 & 0.833 & 17 & 27.53 & 0.811 & 16 & 26.83 & 0.788 & 15 \\
\hline & PPB-AA & 28.19 & 0.832 & $17+2$ & 27.63 & 0.822 & $16+2$ & 26.82 & 0.813 & $15+1$ \\
\hline & Algorithm 2 & 29.67 & 0.848 & $17+10$ & 28.66 & 0.832 & $16+10$ & 27.66 & 0.817 & $15+10$ \\
\hline \multirow{7}{*}{ Remote 1} & Lee & 26.12 & 0.900 & 0.26 & 25.23 & 0.880 & 0.19 & 24.46 & 0.860 & 0.20 \\
\hline & SRAD & 27.46 & 0.934 & 0.83 & 27.13 & 0.931 & 0.64 & 26.60 & 0.918 & 0.79 \\
\hline & AA-like & 27.98 & 0.939 & 30 & 26.91 & 0.922 & 36 & 25.91 & 0.896 & 47 \\
\hline & Algorithm 1 & 28.67 & 0.952 & 85 & 27.52 & 0.941 & 96 & 26.69 & 0.920 & 99 \\
\hline & PPB & 27.54 & 0.943 & 44 & 26.81 & 0.929 & 42 & 26.07 & 0.912 & 42 \\
\hline & PPB-AA & 28.03 & 0.935 & $44+9$ & 27.22 & 0.923 & $42+8$ & 26.39 & 0.898 & $42+12$ \\
\hline & Algorithm 2 & 28.79 & 0.953 & $44+35$ & 27.90 & 0.937 & $42+39$ & 26.86 & 0.922 & $42+38$ \\
\hline \multirow{7}{*}{ Remote2 } & Lee & 26.12 & 0.836 & 0.22 & 25.39 & 0.813 & 0.31 & 24.68 & 0.785 & 0.25 \\
\hline & SRAD & 26.93 & 0.865 & 1.03 & 26.59 & 0.846 & 1.42 & 26.30 & 0.832 & 1.34 \\
\hline & AA-like & 27.81 & 0.871 & 50 & 26.84 & 0.844 & 64 & 25.91 & 0.814 & 75 \\
\hline & Algorithm 1 & 28.43 & 0.885 & 143 & 27.57 & 0.866 & 131 & 26.64 & 0.839 & 150 \\
\hline & $\mathrm{PPB}$ & 27.94 & 0.868 & 64 & 27.22 & 0.844 & 63 & 26.55 & 0.821 & 62 \\
\hline & PPB-AA & 28.02 & 0.858 & $64+7$ & 27.23 & 0.831 & $63+8$ & 26.47 & 0.809 & $62+8$ \\
\hline & Algorithm 2 & 29.02 & 0.889 & $64+47$ & 28.04 & 0.865 & $63+52$ & 27.10 & 0.838 & $62+49$ \\
\hline
\end{tabular}

TABle 2: The parameter values used in the denoising experiment.

\begin{tabular}{|c|c|c|c|c|c|c|c|c|c|c|}
\hline \multirow{2}{*}{ Image } & \multirow{2}{*}{ Method } & \multicolumn{3}{|c|}{$L=10$} & \multicolumn{3}{|c|}{$L=7$} & \multicolumn{3}{|c|}{$L=5$} \\
\hline & & $\lambda$ & $\alpha$ & $\beta$ & $\lambda$ & $\alpha$ & $\beta$ & $\lambda$ & $\alpha$ & $\beta$ \\
\hline \multirow{4}{*}{ Lena } & AA-like & 0.23 & - & - & 0.23 & - & - & 0.23 & - & - \\
\hline & Algorithm 1 & 0.02 & 1.9 & 1 & 0.02 & 1 & 1 & 0.02 & 0.4 & 1.1 \\
\hline & PPB-AA & 0.25 & - & - & 0.25 & - & - & 0.25 & - & - \\
\hline & Algorithm 2 & 0.01 & 0.6 & 1 & 0.01 & 0.3 & 1 & 0.01 & 0.4 & 1.1 \\
\hline \multirow{4}{*}{ Boat } & AA-like & 0.22 & - & - & 0.23 & - & - & 0.23 & - & - \\
\hline & Algorithm 1 & 0.02 & 3.3 & 1 & 0.02 & 1.8 & 1 & 0.02 & 0.9 & 1.1 \\
\hline & PPB-AA & 0.22 & - & - & 0.22 & - & - & 0.22 & - & - \\
\hline & Algorithm 2 & 0.01 & 2.7 & 1 & 0.01 & 1.7 & 1 & 0.01 & 1.4 & 1.1 \\
\hline \multirow{4}{*}{ Cameraman } & AA-like & 0.18 & - & - & 0.19 & - & - & 0.19 & - & - \\
\hline & Algorithm 1 & 0.01 & $1 / 12$ & 1 & 0.02 & 2.2 & 1 & 0.02 & 1.1 & 1.1 \\
\hline & PPB-AA & 0.21 & - & - & 0.21 & - & - & 0.2 & - & - \\
\hline & Algorithm 2 & 0.02 & 7.5 & 1 & 0.02 & 3.9 & 1 & 0.02 & 1.5 & 1.1 \\
\hline \multirow{4}{*}{ Remote1 } & AA-like & 0.17 & - & - & 0.17 & - & - & 0.18 & - & - \\
\hline & Algorithm 1 & 0.01 & $1 / 12$ & 1 & 0.01 & $1 / 12$ & 1 & 0.02 & 1.5 & 1.1 \\
\hline & PPB-AA & 0.17 & - & - & 0.16 & - & - & 0.18 & - & - \\
\hline & Algorithm 2 & 0.01 & 3 & 1 & 0.01 & 0.9 & 1 & 0.01 & 1 & 1.1 \\
\hline \multirow{4}{*}{ Remote2 } & AA-like & 0.17 & - & - & 0.18 & - & - & 0.18 & - & - \\
\hline & Algorithm 1 & 0.01 & 0.1 & 1 & 0.02 & 3 & 1 & 0.02 & 1.6 & 1.1 \\
\hline & PPB-AA & 0.17 & - & - & 0.18 & - & - & 0.18 & - & - \\
\hline & Algorithm 2 & 0.01 & 3.2 & 1 & 0.01 & 2 & 1 & 0.01 & 1.2 & 1.1 \\
\hline
\end{tabular}




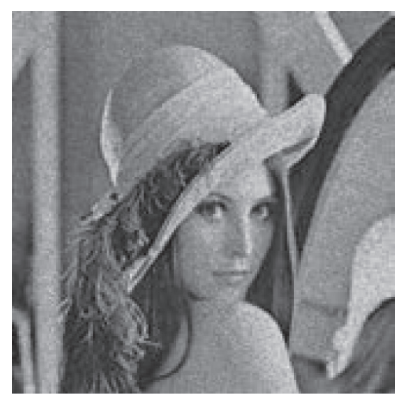

(a)

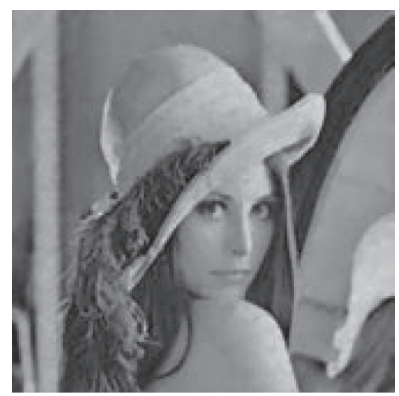

(e)

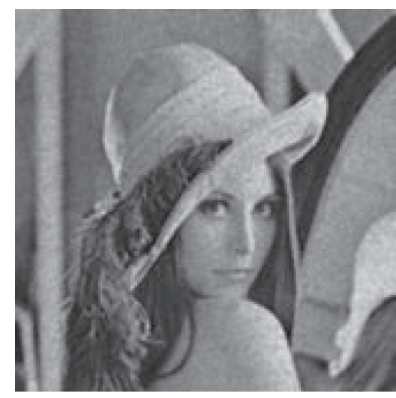

(b)

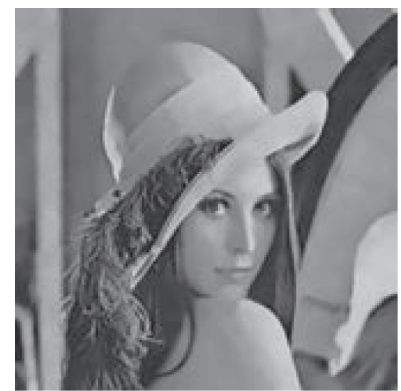

(f)

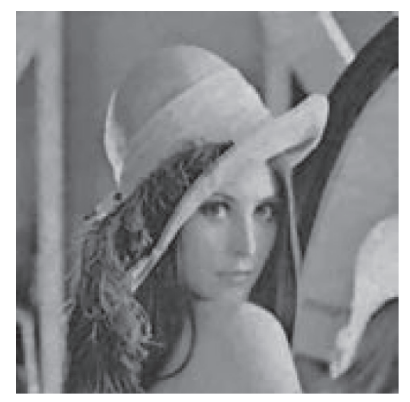

(c)

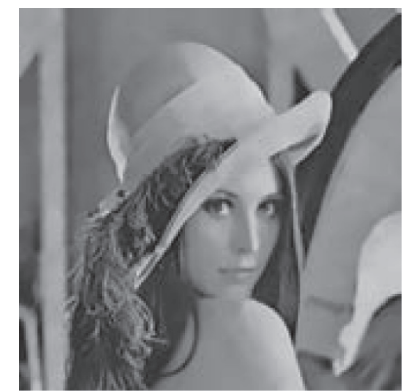

(g)

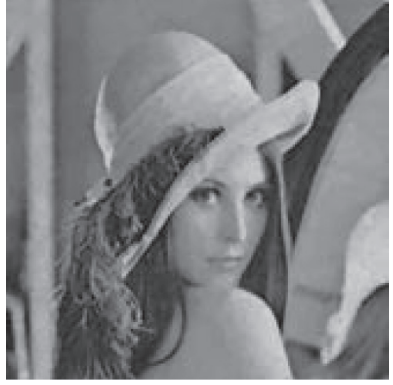

(d)

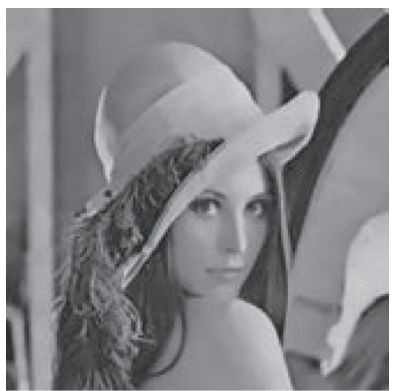

(h)

Figure 3: Denoising results of different methods on Lena image with $L=5$ : (a) PSNR = 18.73dB; (b) PSNR = 27.75 dB; (c) PSNR = 29.02 dB; (d) $P S N R=28.12 \mathrm{~dB}$; (e) PSNR=28.91 dB; (f) PSNR=30.37 dB; (g) PSNR=30.18 dB; (h) PSNR= $30.82 \mathrm{~dB}$.

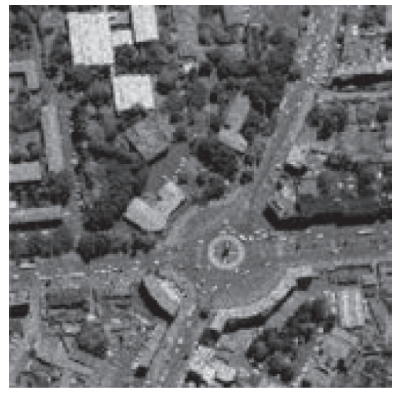

(a)

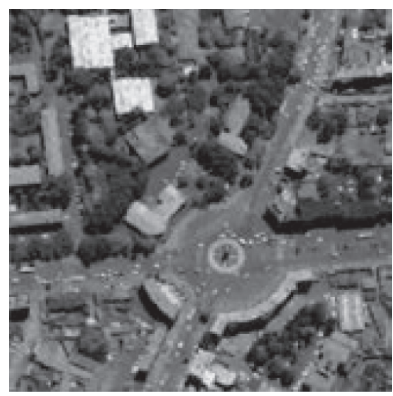

(e)

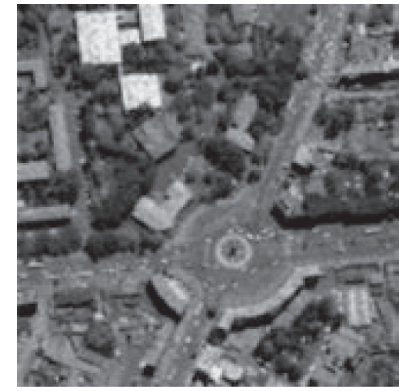

(b)

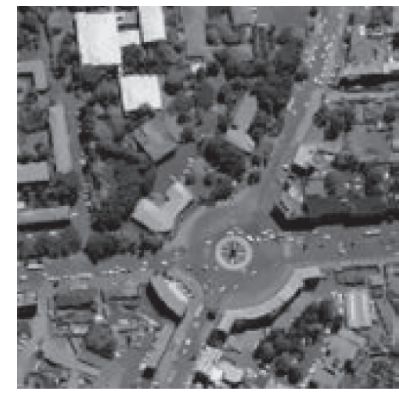

(f)

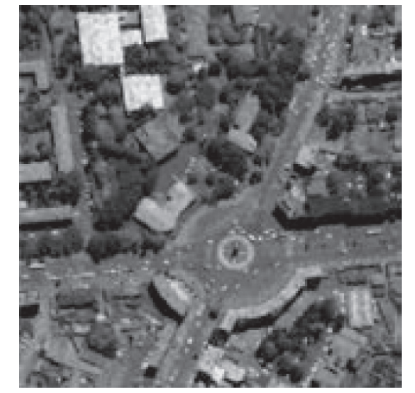

(c)

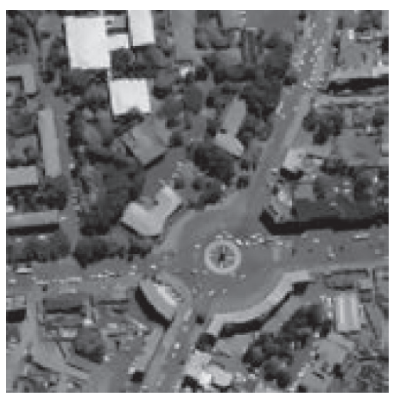

(g)

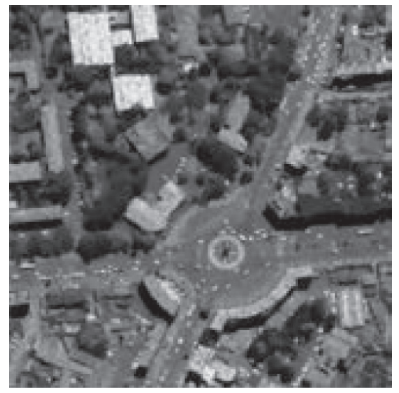

(d)

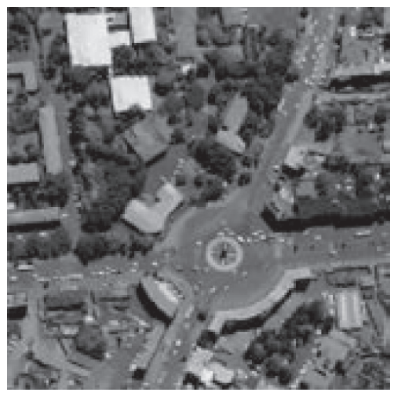

(h)

FiguRE 4: Denoising results of different methods on Remote1 image with $L=5$ : (a) PSNR $=20.44 \mathrm{~dB}$; (b) PSNR $=24.46 \mathrm{~dB}$; (c) $P S N R=26.60 \mathrm{~dB}$; (d) PSNR=25.91 dB; (e) PSNR=26.69 dB; (f) PSNR=26.07 dB; (g) PSNR=26.39 dB; (h) PSNR=26.86 dB.

3.2. Deblurring Experiment. In the second experiment, we compare our proposed method with AA-like method for the simultaneous deblurring and denoising case. The two different blurring operators are the motion blur with length 5 and angle 30 and Gaussian blur with a window size $7 \times 7$ and a standard deviation of 2 . And after the test images are blurred, they are corrupted by multiplicative Nakagami noise with $L=10, L=7$, and $L=5$, respectively. We list the parameter values in Table 3 , and the empirical choice of $\beta$ is the same as denoising case. The numerical results are presented in Table 4 . We can see that the PSNR and SSIM values of the restored images by Algorithm 1 are higher than that of 


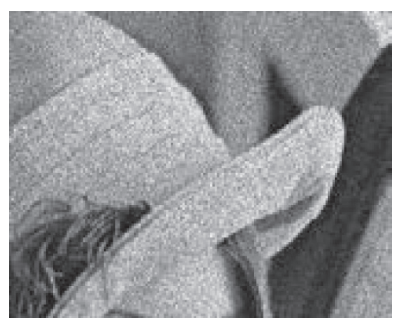

(a)

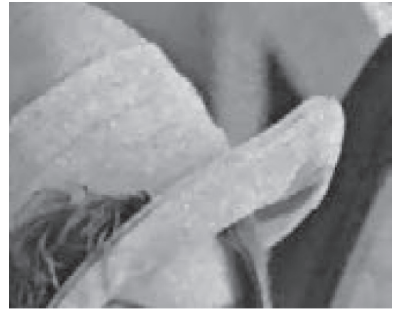

(e)

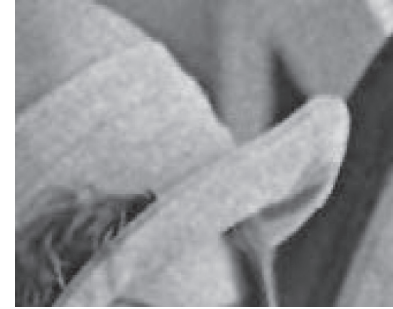

(b)

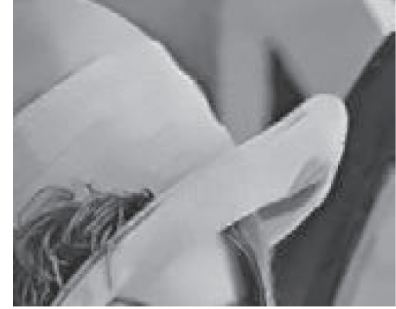

(f)

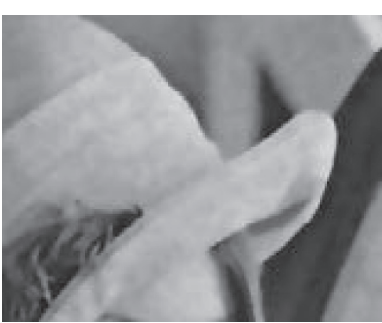

(c)

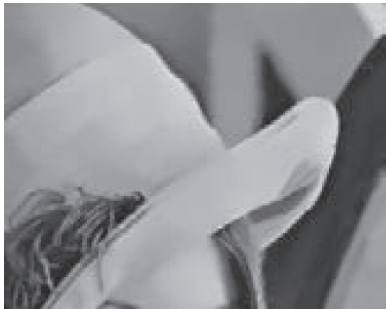

(g)

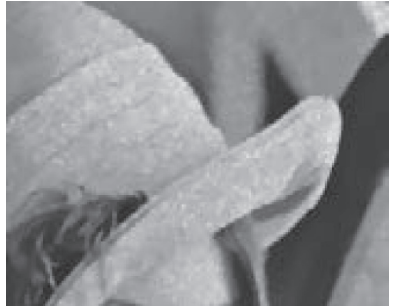

(d)

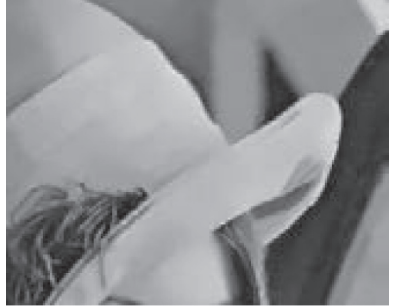

(h)

Figure 5: Partial enlarged detail of the denoising results on Lena image with $L=10$ : (a) PSNR $=21.73 \mathrm{~dB}$; (b) PSNR=29.52 dB; (c) PSNR=29.75 dB; (d) PSNR=30.01 dB; (e) PSNR=30.99 dB; (f) PSNR= $31.82 \mathrm{~dB}$; (g) PSNR= $32.05 \mathrm{~dB}$; (h) PSNR=32.28 dB.

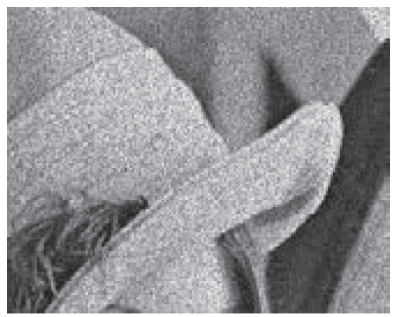

(a)

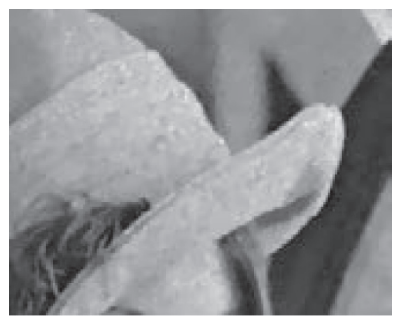

(e)

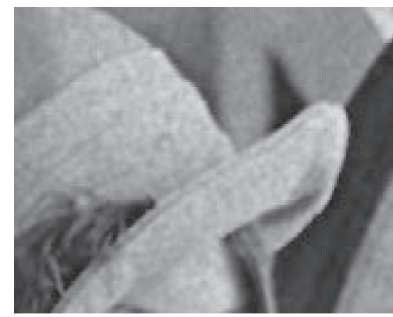

(b)

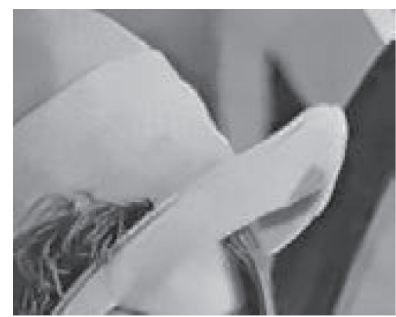

(f)

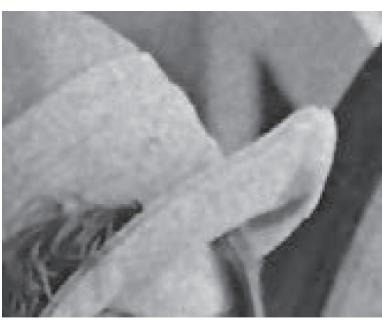

(c)

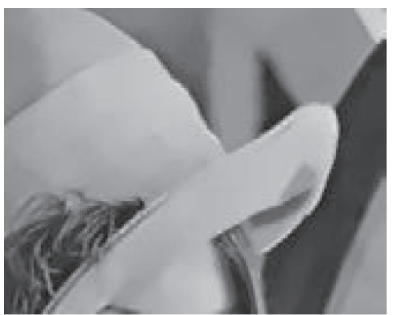

(g)

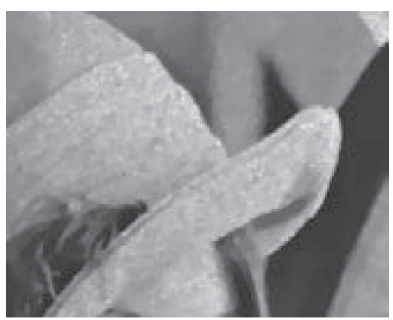

(d)

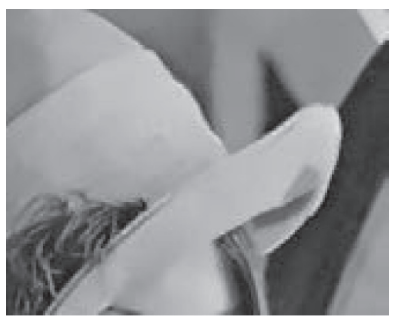

(h)

FIGURE 6: Partial enlarged detail of the denoising results on Lena image with $L=7$ : (a) $P S N R=20.16 \mathrm{~dB}$; (b) $P S N R=28.58 \mathrm{~dB}$; (c) $P S N R=29.42 \mathrm{~dB}$; (d) PSNR=29.01 dB; (e) PSNR=29.95 dB; (f) PSNR=31.08 dB; (g) PSNR=31.09 dB; (h) PSNR=31.29 dB.

the AA-like method. Moreover, we show the degraded images and the restored results of both methods for the images "Cameraman" in Figures 11-13. One can see Algorithm 1 preserves more details (e.g., the tripod in "Cameraman"). Due to oversmoothing, the AA-like method tends to lose details and attenuate the image contrast. Note that both methods cost more time than that in the denoising case because of the existence of the blurring operator.

\subsection{Real SAR Images Restoration Experiment.} Furthermore, we evaluate the performance of a newly proposed model for despeckling based on three real SAR images. The stopping criterion is the same as that of the denoising experiment. Since there are no original noise-free images to be compared, PSNR and SSIM cannot be used to evaluate the quality of recovered images. Assuming $L=5$, we tune the parameters until the recovered images show the best results. The parameters settings are reported in Table 5 . Figures 14-16 present the denoising results on real SAR images. It is obvious that all of their objective function values are monotonically decreasing. The less speckle is eliminated by Lee filter, SRAD method, and AA-like method. Lee filter does not enhance edges. There are staircasing effects existing in the results of AA-like method. Algorithm 1 can remove noise more effectively than AA-like method. PPB method 


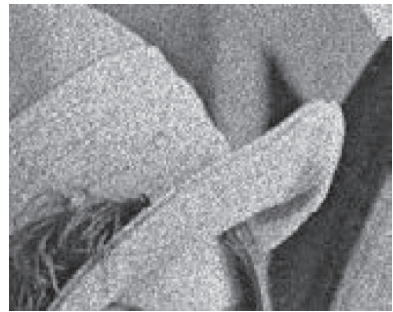

(a)

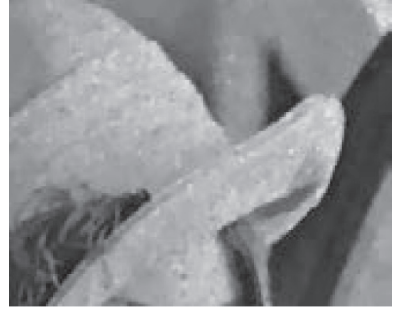

(e)

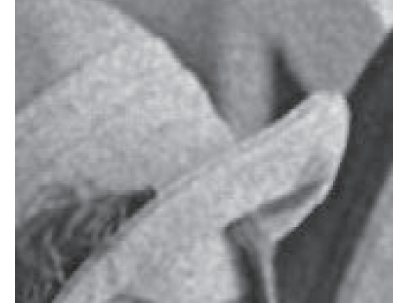

(b)

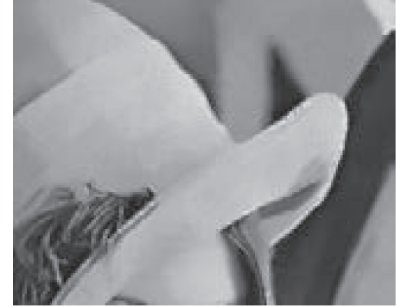

(f)

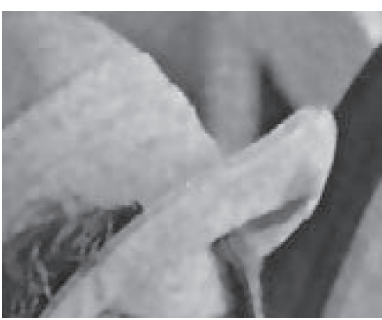

(c)

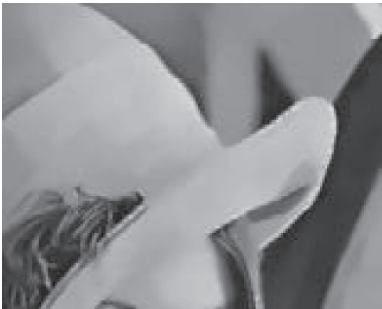

(g)

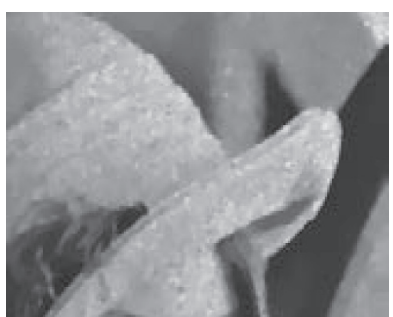

(d)

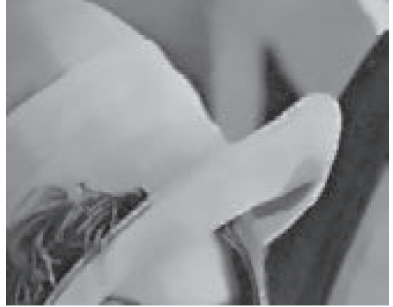

(h)

FIgURE 7: Partial enlarged detail of the denoising results on Lena image with $L=5$ : (a) PSNR $=18.73 \mathrm{~dB}$; (b) PSNR=27.75 dB; (c) PSNR=29.02 dB; (d) PSNR=28.12 dB; (e) PSNR=28.91 dB; (f) PSNR=30.37 dB; (g) PSNR= 30.18 dB; (h) PSNR=30.82 dB.

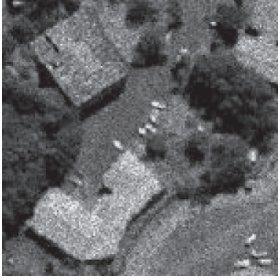

(a)

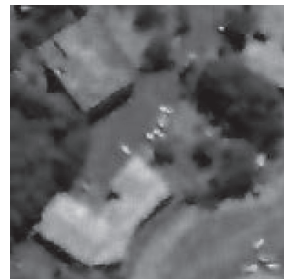

(b)

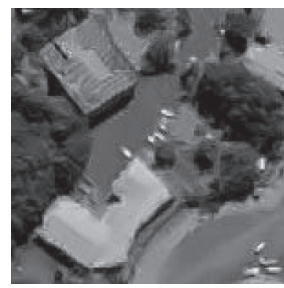

(f)

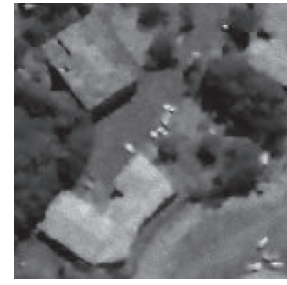

(c)

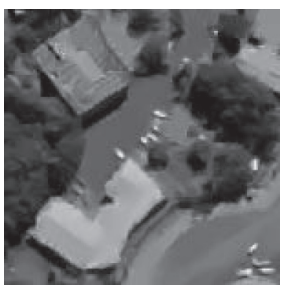

(g)

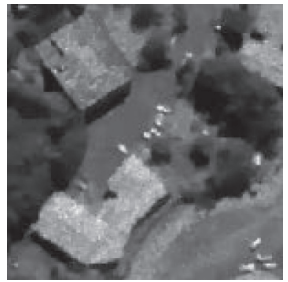

(d)

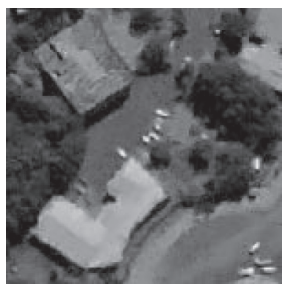

(h)

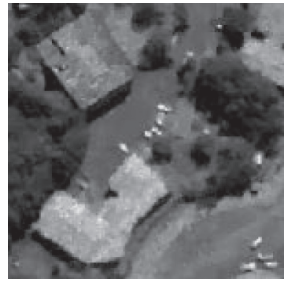

(e)

Figure 8: Partial enlarged detail of the denoising results on Remote1 image with $L=10$ : (a) PSNR $=23.45 \mathrm{~dB}$; (b) PSNR $=26.12 \mathrm{~dB}$; (c) $P S N R=27.46 \mathrm{~dB}$; (d) PSNR=27.98 dB; (e) PSNR=28.67 dB; (f) PSNR=27.54 dB; (g) PSNR=28.03 dB; (h) PSNR=28.79 dB.

performs well in restoring images, such as smoother regions and better shape preservation. But there are some spurious artifacts in the edge of the images. Comparing the experimental results, we can find that Algorithm 2 reduces the artifacts more effectively than PPB-AA method. At the same time, the staircasing effects also decrease in these two methods.

3.4. The Influence of Parameters and Mean. Here, we study the sensitivities of parameters and the effect of mean of our restoration model. According to the analysis in Section 2.1, the value of parameter $\beta$ varies with the level of the noise. Experimentally, $\beta$ is chosen as 1 when $L>5$. When $L=5, \beta$ is set as 1.1. In the proposed model, parameter $\alpha$ needs to satisfy condition $\alpha \geq(1 / 12)$. Parameter $\lambda$ is set as $\lambda^{2}<(1 / 72)$ based on the description in Section 2.3, which is changing from 0.01 to 0.11 with an interval 0.01 in experiment. In the denoising case, we take the noisy images "Lena" and "Cameraman" as examples. For each image, we vary the values of $\alpha$ with three levels of the noise, $L=10,7,5$. The PSNR values of restored images using Algorithm 1 are presented in Figure 17. We observe that there is an upmost point on each curve. With the increasing $\alpha$, the performance 


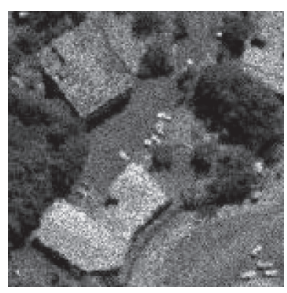

(a)

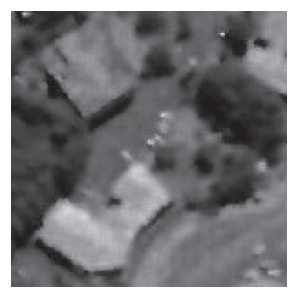

(b)

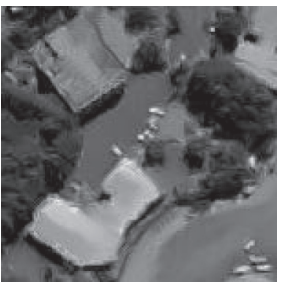

(f)

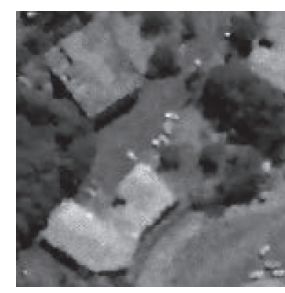

(c)

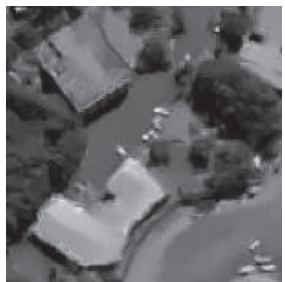

(g)

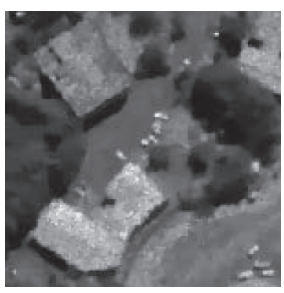

(d)

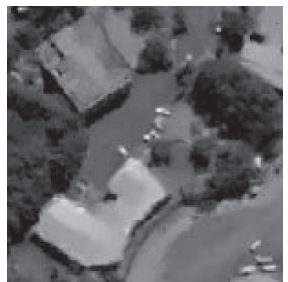

(h)

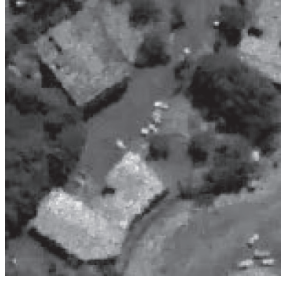

(e)

Figure 9: Partial enlarged detail of the denoising results on Remotel image with $L=7$ : (a) PSNR $=21.90 \mathrm{~dB}$; (b) PSNR $=25.23 \mathrm{~dB}$; (c) $P S N R=27.13 \mathrm{~dB}$; (d) PSNR = 26.91 dB; (e) PSNR=27.52 dB; (f) PSNR=26.81 dB; (g) PSNR=27.22 dB; (h) PSNR=27.90 dB.

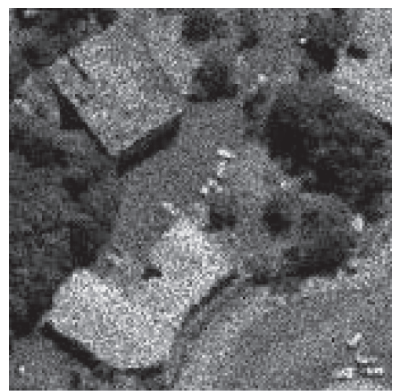

(a)

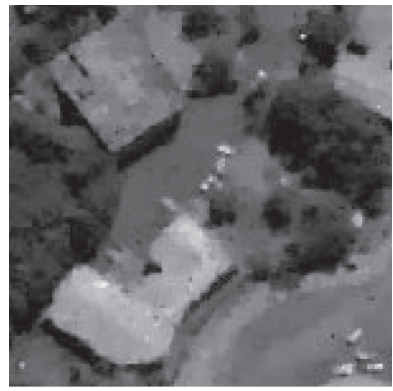

(e)

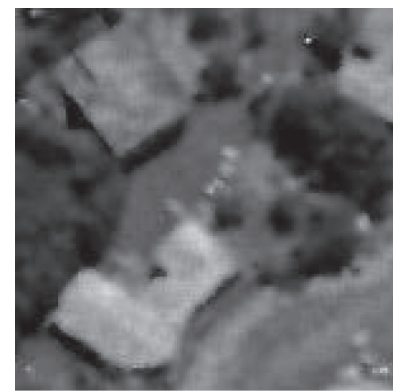

(b)

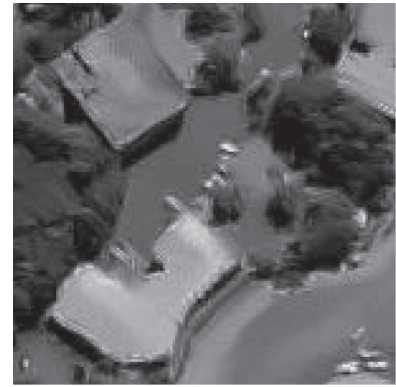

(f)

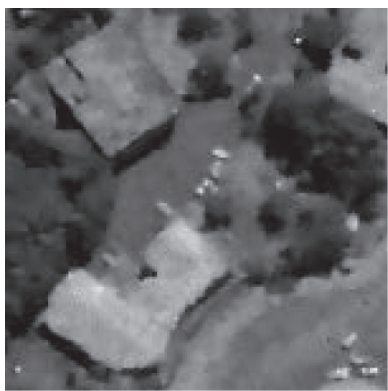

(c)

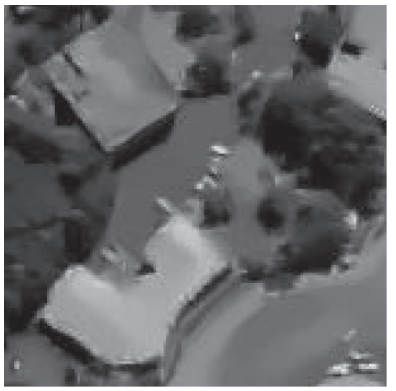

(g)

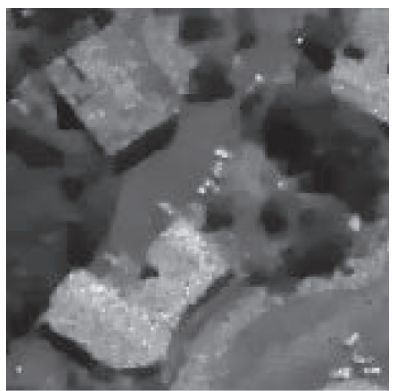

(d)

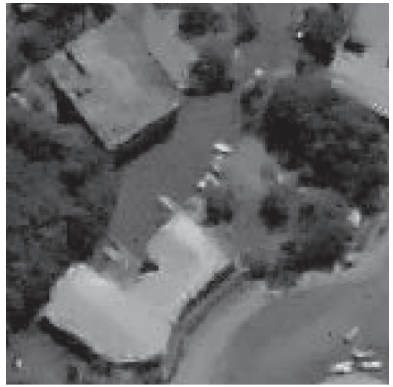

(h)

Figure 10: Partial enlarged detail of the denoising results on Remote1 image with $L=5$ : (a) PSNR $=20.44 \mathrm{~dB}$; (b) PSNR $=24.46 \mathrm{~dB}$; (c) $P S N R=26.60 \mathrm{~dB}$; (d) PSNR=25.91 dB; (e) PSNR=26.69 dB; (f) PSNR=26.07 dB; (g) PSNR=26.39 dB; (h) PSNR=26.86 dB.

of "Lena" with $L=5$ and "Cameraman" with $L=10$ decreases rapidly, which means that the denoising results are sensitive to value of $\alpha$ for each $L$. In the simultaneous deblurring and denoising case, we take the noisy image of "Cameraman" blurred by the motion blur with length 5 and angle 30 and then corrupted by multiplicative Nakagami noise with $L=10$ as an example. The parameter settings are $\lambda=0.01, \alpha=7$, and $\beta=1$ in this case known from Table 3 . At first, for the case of $\lambda=0.01$, we study the sensitivity of the parameter $\alpha$ in terms of solution quality and computation time. The PSNR (dB), SSIM, and CPU time (s) used for various values of $\alpha$ are listed in Table 6. We observe that if $\alpha$ is less than 7, the PSNR value of the restored image by Algorithm 1 increases with the increase in $\alpha$. However, the PSNR and SSIM values decrease as the value of $\alpha$ increases when $\alpha$ is more than 7. The numerical results indicate that there is a certain value of $\alpha$ to make the model get the best quality of recovered image for a fixed value of $\lambda$. Then, for the case of $\alpha=7$, we further analyze the sensitivity of the parameter $\lambda$. The PSNR (dB), SSIM, and CPU time (s) used 
TABle 3: The parameter values used in deblurring experiment.

\begin{tabular}{|c|c|c|c|c|c|c|c|c|}
\hline \multirow{2}{*}{$L$} & \multirow{2}{*}{ Image } & \multirow{2}{*}{ Method } & \multicolumn{3}{|c|}{ Motion blur } & \multicolumn{3}{|c|}{ Gaussian blur } \\
\hline & & & $\lambda$ & $\alpha$ & $\beta$ & $\lambda$ & $\alpha$ & $\beta$ \\
\hline \multirow{6}{*}{10} & \multirow{2}{*}{ Lena } & AA-like & 0.03 & - & - & 0.04 & - & - \\
\hline & & Algorithm 1 & 0.01 & 3.5 & 1 & 0.01 & 7.1 & 1 \\
\hline & \multirow{2}{*}{ Boat } & AA-like & 0.04 & - & - & 0.04 & - & - \\
\hline & & Algorithm 1 & 0.01 & 5.5 & 1 & 0.01 & 11.5 & 1 \\
\hline & \multirow{2}{*}{ Cameraman } & AA-like & 0.03 & - & - & 0.03 & - & - \\
\hline & & Algorithm 1 & 0.01 & 7 & 1 & 0.01 & 12 & 1 \\
\hline \multirow{6}{*}{7} & \multirow{2}{*}{ Lena } & AA-like & 0.03 & - & - & 0.04 & - & - \\
\hline & & Algorithm 1 & 0.01 & 1.9 & 1 & 0.01 & 4 & 1 \\
\hline & \multirow{2}{*}{ Boat } & AA-like & 0.04 & - & - & 0.04 & - & - \\
\hline & & Algorithm 1 & 0.01 & 3.2 & 1 & 0.01 & 6.2 & 1 \\
\hline & \multirow{2}{*}{ Cameraman } & AA-like & 0.03 & - & - & 0.03 & - & - \\
\hline & & Algorithm 1 & 0.01 & 3.6 & 1 & 0.01 & 7 & 1 \\
\hline \multirow{6}{*}{5} & \multirow{2}{*}{ Lena } & AA-like & 0.03 & - & - & 0.04 & - & - \\
\hline & & Algorithm 1 & 0.01 & 1 & 1.1 & 0.01 & 2 & 1.1 \\
\hline & \multirow{2}{*}{ Boat } & AA-like & 0.03 & - & - & 0.04 & - & - \\
\hline & & Algorithm 1 & 0.01 & 1.8 & 1.1 & 0.01 & 3.4 & 1.1 \\
\hline & \multirow{2}{*}{ Cameraman } & AA-like & 0.03 & - & - & 0.03 & - & - \\
\hline & & Algorithm 1 & 0.01 & 1.8 & 1.1 & 0.01 & 3.7 & 1.1 \\
\hline
\end{tabular}

TABLE 4: The comparison of different deblurring and denoising methods.

\begin{tabular}{|c|c|c|c|c|c|c|c|c|}
\hline \multirow{2}{*}{$L$} & \multirow{2}{*}{ Image } & \multirow{2}{*}{ Method } & \multicolumn{3}{|c|}{ Motion blur } & \multicolumn{3}{|c|}{ Gaussian blur } \\
\hline & & & PSNR (dB) & SSIM & Time (s) & PSNR (dB) & SSIM & Time (s) \\
\hline \multirow{6}{*}{10} & \multirow{2}{*}{ Lena } & AA-like & 27.73 & 0.832 & 522 & 26.80 & 0.828 & 554 \\
\hline & & Algorithm 1 & 29.40 & 0.903 & 941 & 28.29 & 0.874 & 869 \\
\hline & \multirow{2}{*}{ Boat } & AA-like & 25.50 & 0.804 & 587 & 24.47 & 0.751 & 518 \\
\hline & & Algorithm 1 & 26.48 & 0.840 & 2302 & 25.54 & 0.796 & 2349 \\
\hline & \multirow{2}{*}{ Cameraman } & AA-like & 23.58 & 0.674 & 88 & 22.21 & 0.614 & 97 \\
\hline & & Algorithm 1 & 25.21 & 0.750 & 486 & 23.57 & 0.724 & 638 \\
\hline \multirow{6}{*}{7} & \multirow{2}{*}{ Lena } & AA-like & 27.03 & 0.806 & 603 & 26.47 & 0.815 & 713 \\
\hline & & Algorithm 1 & 28.76 & 0.890 & 1042 & 27.84 & 0.863 & 1012 \\
\hline & \multirow{2}{*}{ Boat } & AA-like & 25.03 & 0.777 & 778 & 24.17 & 0.731 & 648 \\
\hline & & Algorithm 1 & 25.96 & 0.818 & 2210 & 25.06 & 0.769 & 2215 \\
\hline & \multirow{2}{*}{ Cameraman } & AA-like & 23.31 & 0.649 & 120 & 22.09 & 0.594 & 120 \\
\hline & & Algorithm 1 & 24.66 & 0.748 & 460 & 23.37 & 0.716 & 491 \\
\hline \multirow{6}{*}{5} & \multirow{2}{*}{ Lena } & AA-like & 26.34 & 0.778 & 738 & 25.96 & 0.805 & 991 \\
\hline & & Algorithm 1 & 27.99 & 0.874 & 1164 & 27.15 & 0.847 & 1131 \\
\hline & \multirow{2}{*}{ Boat } & AA-like & 24.51 & 0.741 & 708 & 23.80 & 0.710 & 922 \\
\hline & & Algorithm 1 & 25.43 & 0.798 & 2198 & 24.56 & 0.748 & 2319 \\
\hline & \multirow{2}{*}{ Cameraman } & AA-like & 22.99 & 0.619 & 142 & 21.81 & 0.575 & 143 \\
\hline & & Algorithm 1 & 24.25 & 0.736 & 528 & 22.97 & 0.709 & 562 \\
\hline
\end{tabular}

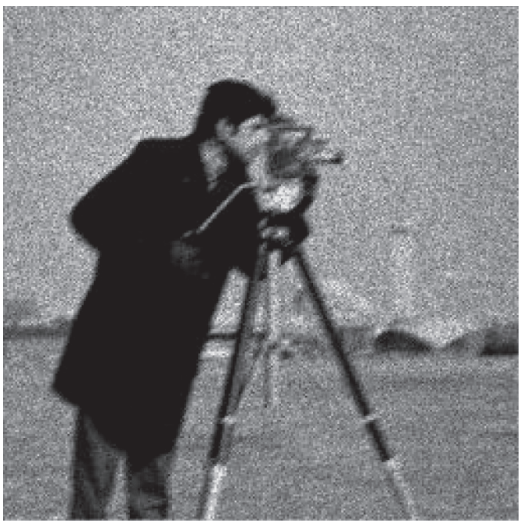

(a)

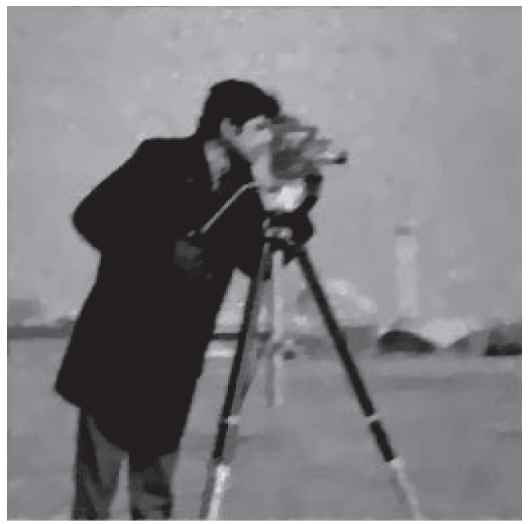

(b)

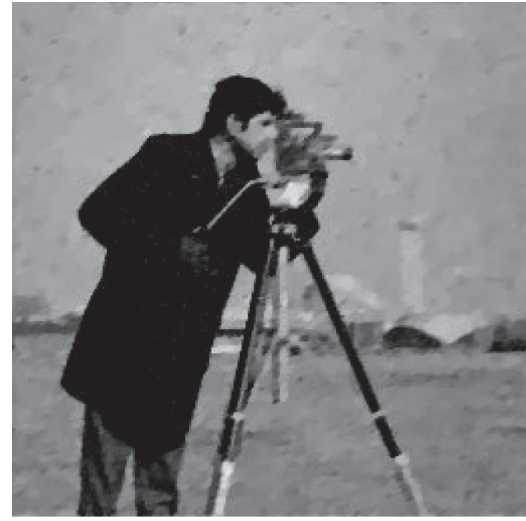

(c)

Figure 11: Continued. 


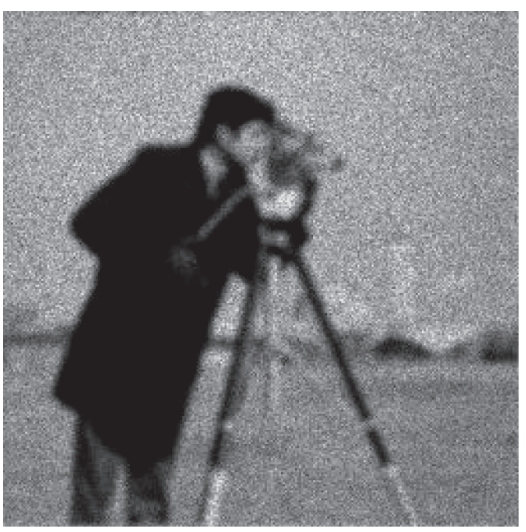

(d)

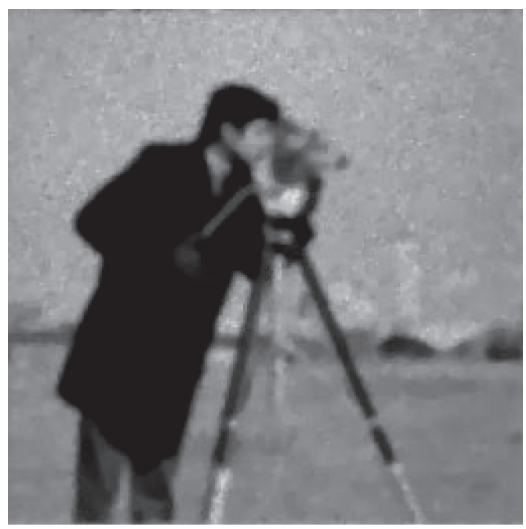

(e)

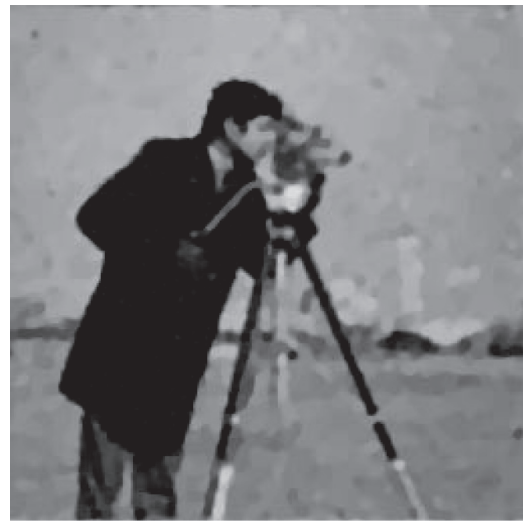

(f)

Figure 11: Deblurring results of different methods on Cameraman image with $L=10$ : (a) degraded (motion); (b) AA-like (23.58 dB); (c) Algorithm 1 (25.21 dB); (d) degraded (Gaussian); (e) AA-like (22.21 dB); (f) Algorithm 1 (23.57 dB).

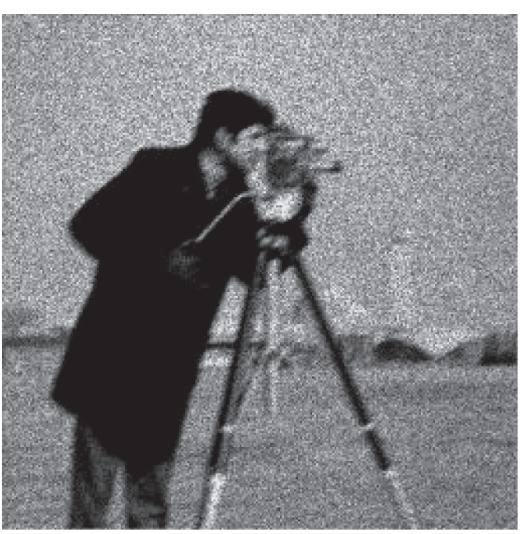

(a)

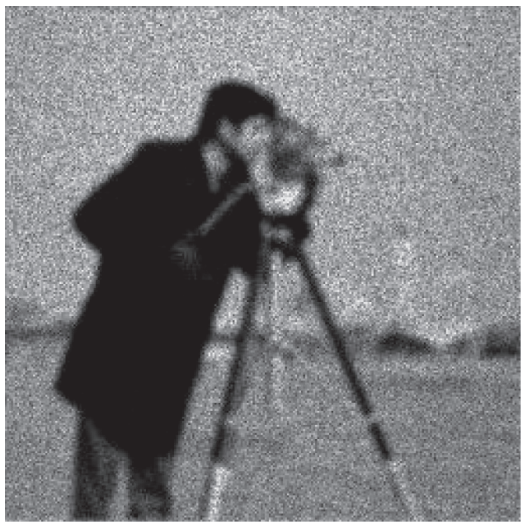

(d)

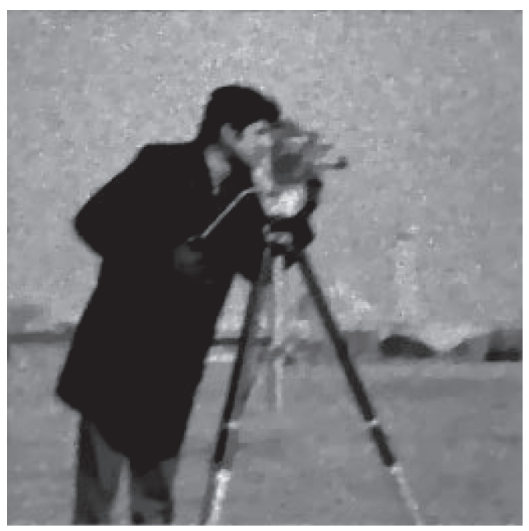

(b)

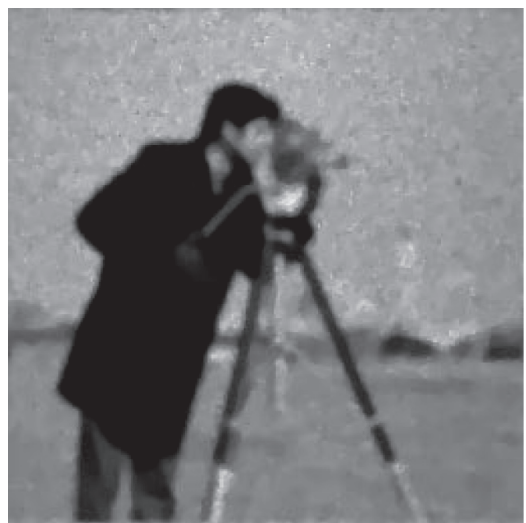

(e)

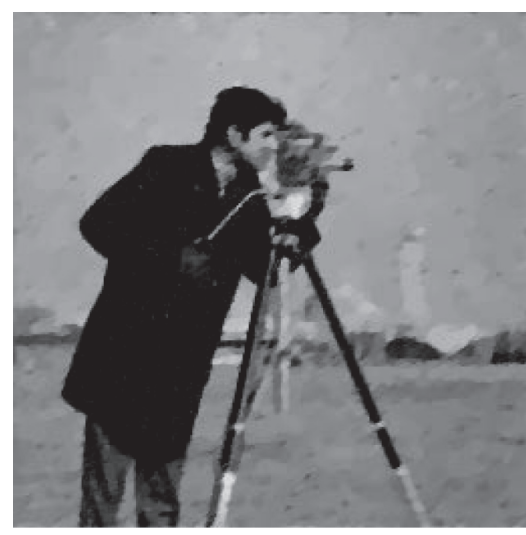

(c)

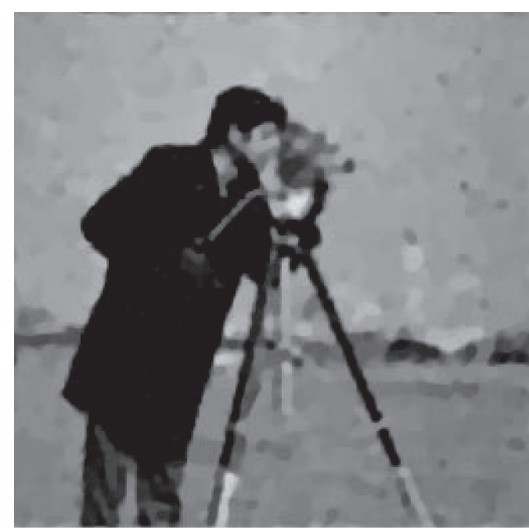

(f)

Figure 12: Deblurring results of different methods on Cameraman image with $L=7$ : (a) degraded (motion); (b) AA-like (23.31 dB); (c) Algorithm 1 (24.66 dB); (d) degraded (Gaussian); (e) AA-like (22.09 dB); (f) Algorithm 1 (23.37 dB).

for various values of $\lambda$ are listed in Table 7. From Table 7, we observe that $\lambda=0.01$ is the best choice for obtaining the highest PSNR value of denoised image.

Finally, in order to discuss the influence of preserving the mean of the original image, there are four assumptions, including recovered images obtained from model (9) and model (9) with preserving 1 times, 1.2 times, 0.8 times the mean of the original image, respectively. Table 8 reports the PSNR (dB) and SSIM values on Cameraman image with different mean. We find the fact the PSNR $(\mathrm{dB})$ and SSIM 


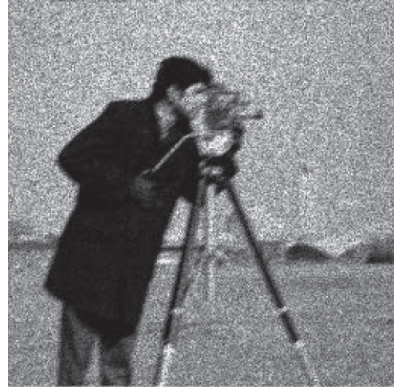

(a)

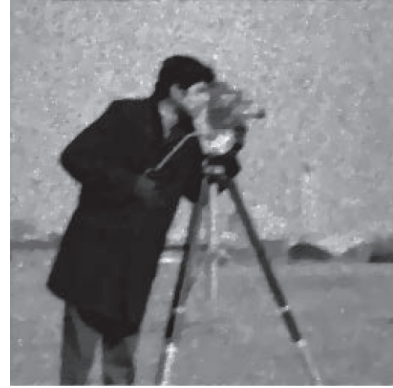

(b)

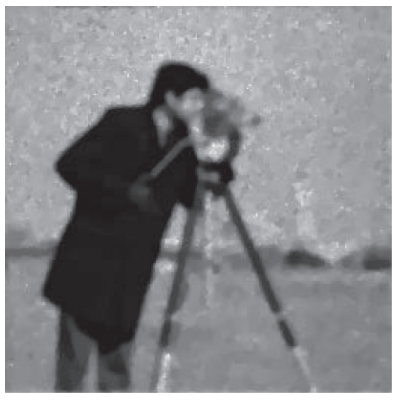

(e)

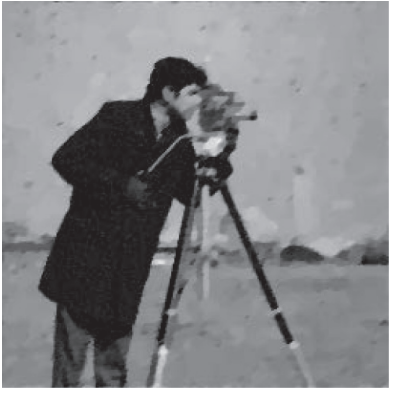

(c)

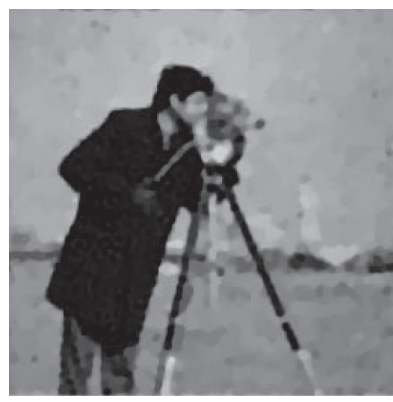

(f)

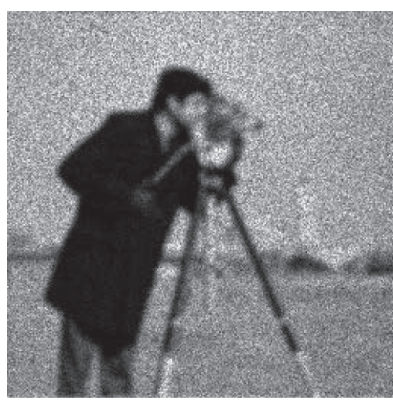

(d)

FiguRE 13: Deblurring results of different methods on Cameraman image with $L=5$ : (a) degraded (motion); (b) AA-like (22.99 dB); (c) Algorithm 1 (24.25 dB); (d) degraded (Gaussian); (e) AA-like (21.81 dB); (f) Algorithm 1 (22.97 dB).

TABLE 5: The parameter values used in SAR images restoration experiment.

\begin{tabular}{|c|c|c|c|c|}
\hline Images & Methods & $\lambda$ & $\alpha$ & $\beta$ \\
\hline \multirow{4}{*}{ SAR image 1} & AA-like & 0.12 & - & - \\
\hline & Algorithm 1 & 0.02 & $1 / 12$ & 1.1 \\
\hline & PPB-AA & 0.12 & - & - \\
\hline & Algorithm 2 & 0.02 & 0.1 & 1.1 \\
\hline \multirow{4}{*}{ SAR image 2} & AA-like & 0.15 & - & - \\
\hline & Algorithm 1 & 0.02 & $1 / 12$ & 1.1 \\
\hline & PPB-AA & 0.17 & - & - \\
\hline & Algorithm 2 & 0.01 & $1 / 12$ & 1.1 \\
\hline \multirow{4}{*}{ SAR image 3} & AA-like & 0.15 & - & - \\
\hline & Algorithm 1 & 0.02 & $1 / 12$ & 1.1 \\
\hline & PPB-AA & 0.15 & - & - \\
\hline & Algorithm 2 & 0.02 & $1 / 12$ & 1.1 \\
\hline
\end{tabular}

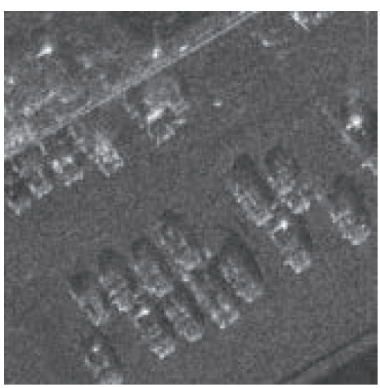

(a)

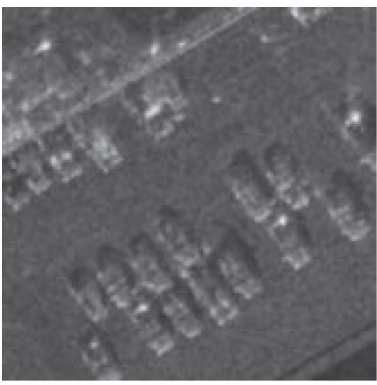

(b)

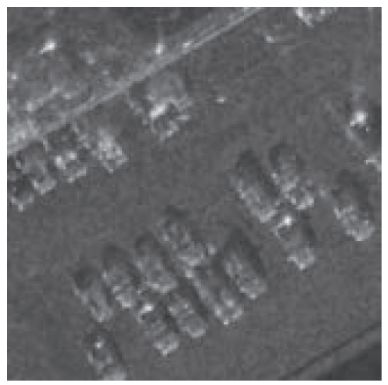

(c)

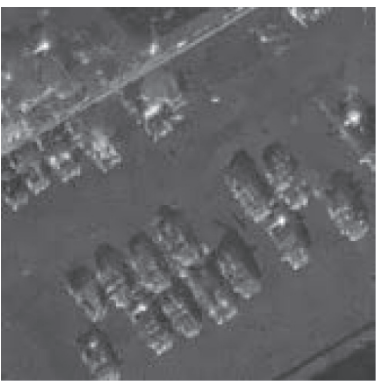

(d)

Figure 14: Continued. 

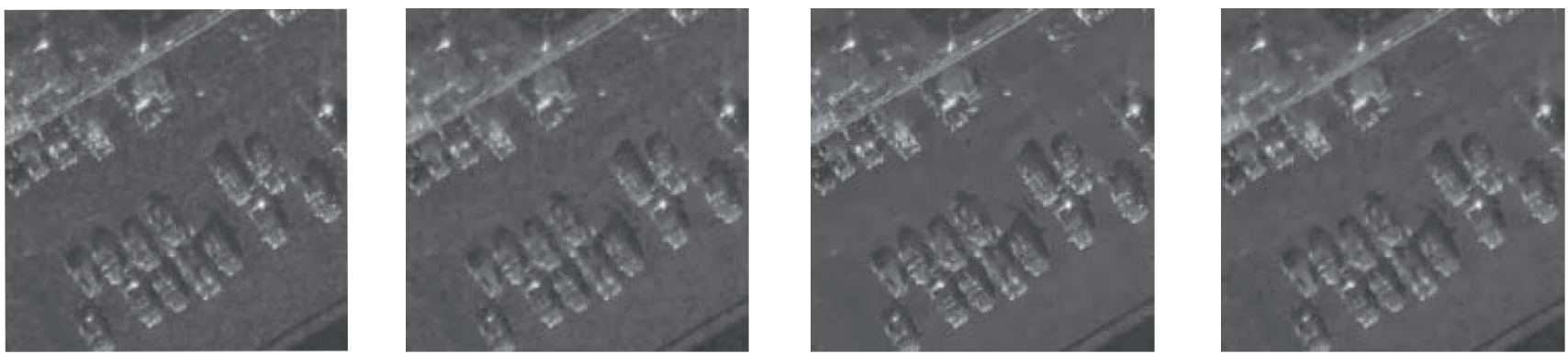

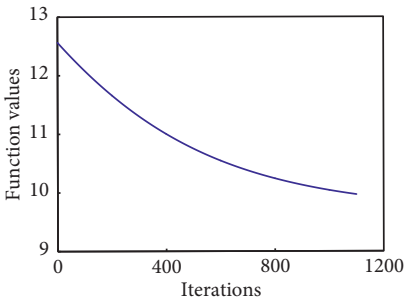

(e)

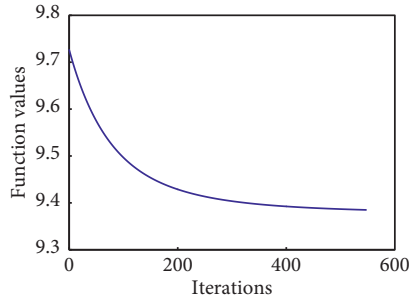

(f)

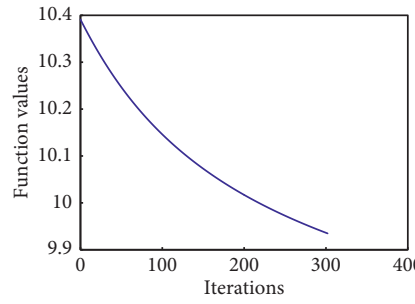

(g)

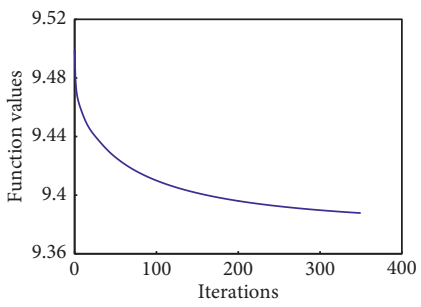

(h)

FIGURe 14: Denoising results on SAR image 1: (a) degraded image; (b) Lee; (c) SRAD; (d) PPB; (e) AA-like; (f) Algorithm 1; (g) PPB-AA; (h) Algorithm 2.

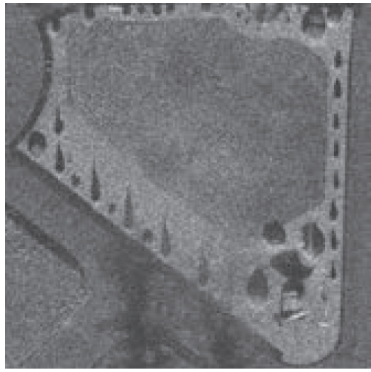

(a)
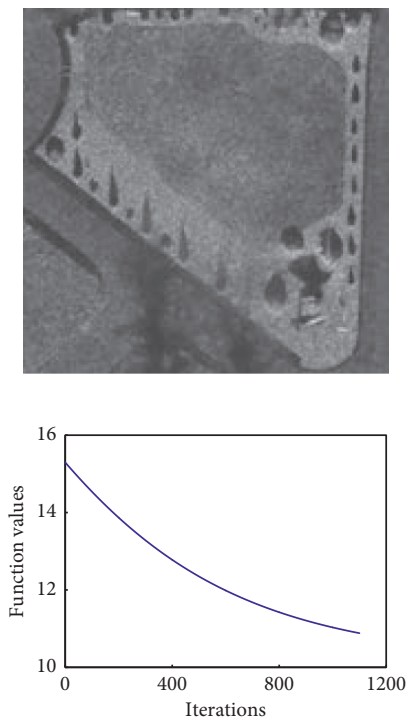

(e)

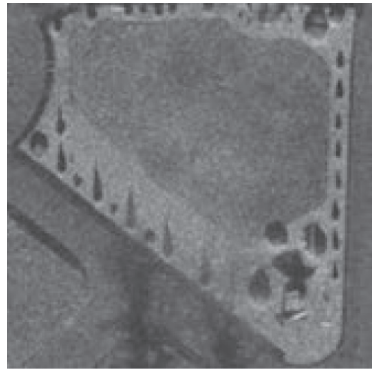

(b)
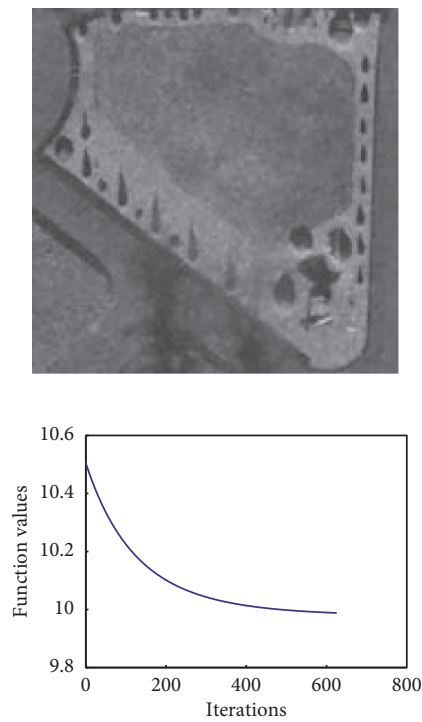

(f)

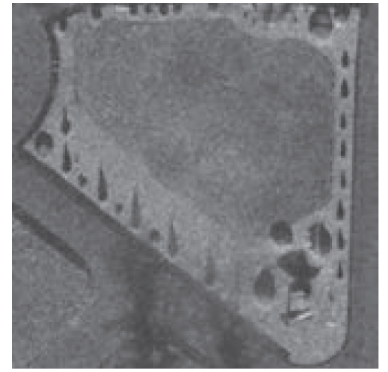

(c)
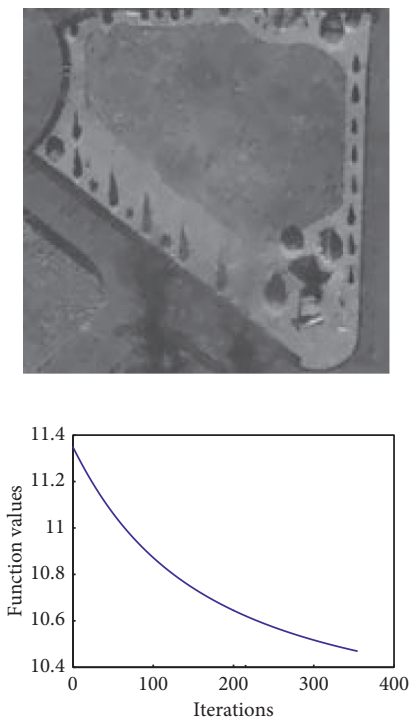

(g)

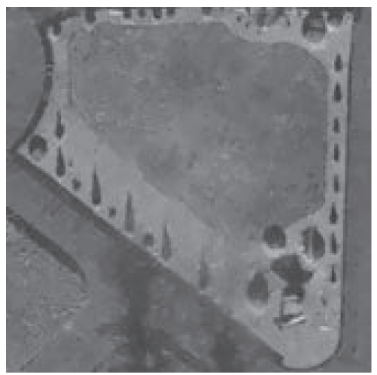

(d)
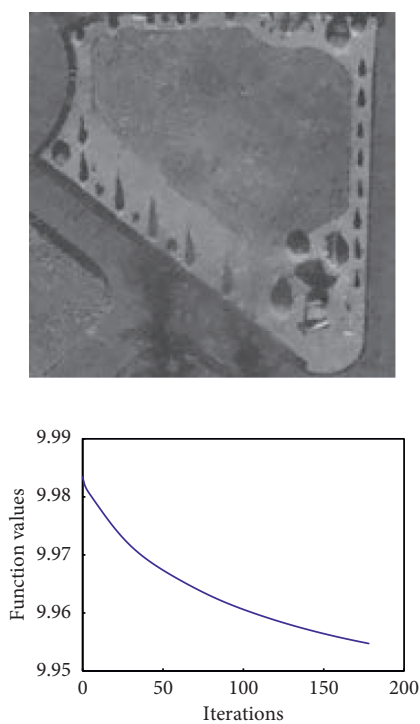

(h)

FIGURE 15: Denoising results on SAR image 2: (a) degraded image; (b) Lee; (c) SRAD; (d) PPB; (e) AA-like; (f) Algorithm 1; (g) PPB-AA; (h) Algorithm 2. 


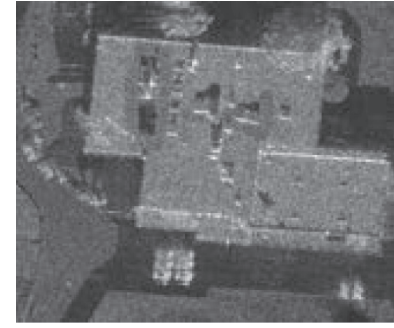

(a)
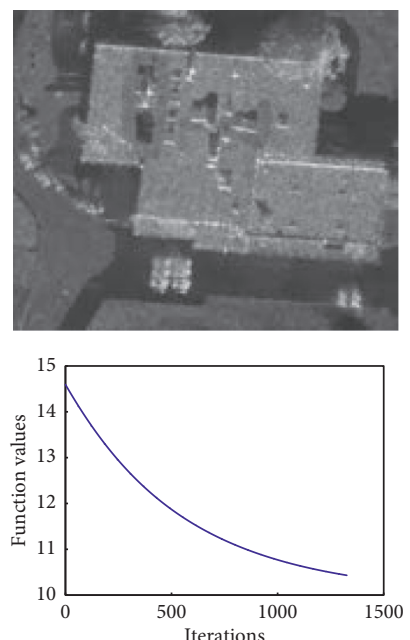

(e)

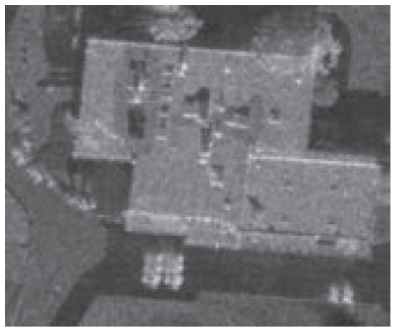

(b)
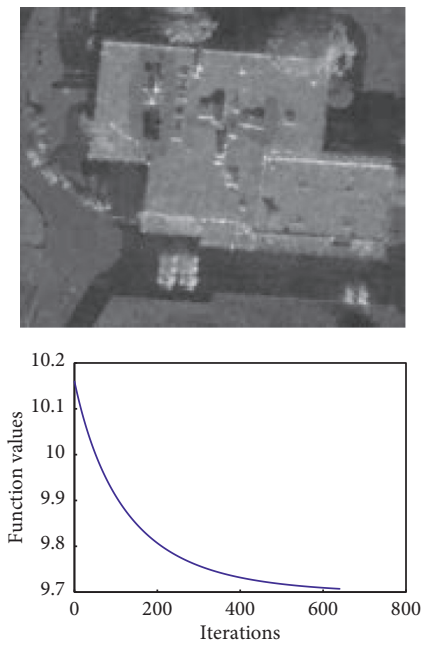

(f)

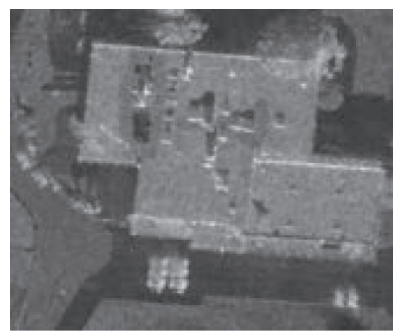

(c)
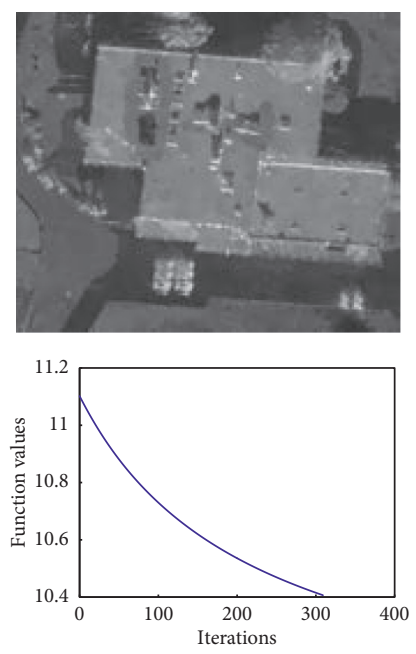

(g)

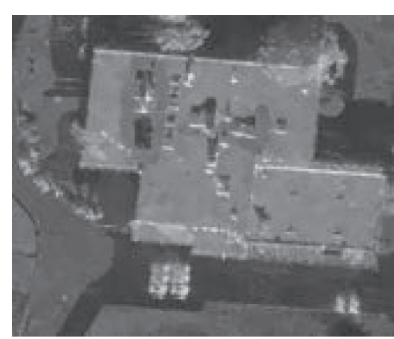

(d)
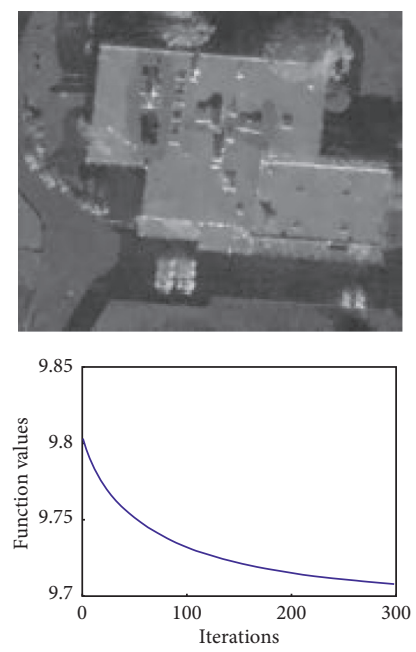

(h)

FIGURE 16: Denoising results on SAR image 3: (a) degraded image; (b) Lee; (c) SRAD; (d) PPB; (e) AA-like; (f) Algorithm 1; (g) PPB-AA; (h) Algorithm 2.

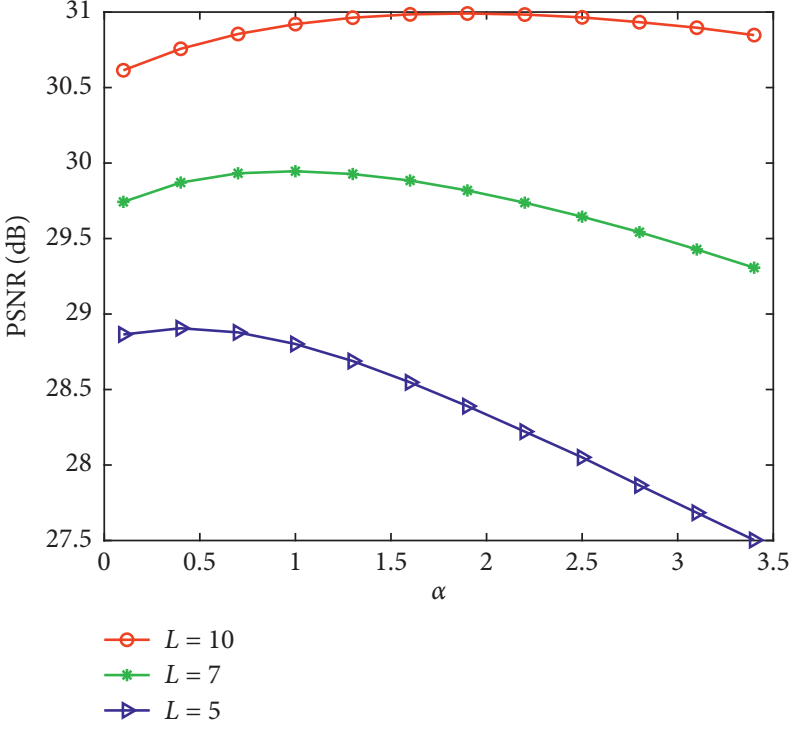

(a)

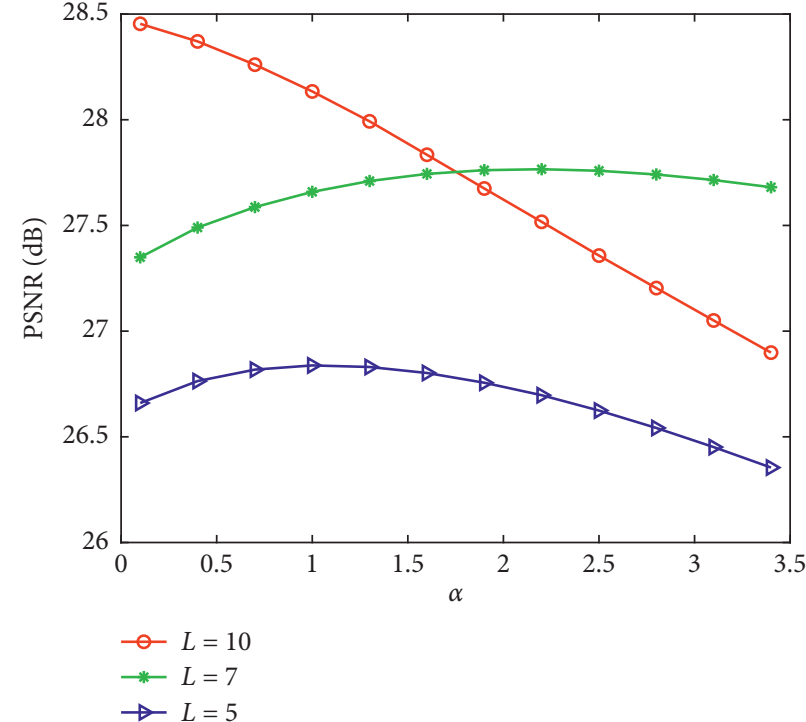

(b)

Figure 17: The PSNR values against the values of $\alpha$ under three noise levels: (a) Lena; (b) Cameraman.

values obtained from model (9) with preserving mean of the original image are almost higher than the others. As shown in Figure 18, when the mean of recovered image is larger than that of the original image, the restored image by Algorithm 1 looks lighter. In contrast, the restored image is darker. PSNR and SSIM values of both decrease significantly. 
TABLE 6: Numerical results of Algorithm 1 with parameter $\lambda=0.01$ on Cameraman image.

\begin{tabular}{lcccrr}
\hline$\alpha$ & 0.1 & 3 & 7 & 8 & 9 \\
\hline PSNR (dB) & 24.15 & 24.99 & $\mathbf{2 5 . 2 1}$ & 25.20 & 25.17 \\
SSIM & 0.762 & 0.776 & 0.750 & 0.738 & 5.726 \\
Time (s) & 274 & 395 & 486 & 528 \\
\hline
\end{tabular}

TABLe 7: Numerical results of Algorithm 1 with parameter $\alpha=7$ on Cameraman image.

\begin{tabular}{lccccc}
\hline$\lambda$ & 0.01 & 0.02 & 0.05 & 0.08 & 0.11 \\
\hline PSNR (dB) & $\mathbf{2 5 . 2 1}$ & 24.85 & 23.77 & 23.10 & 22.62 \\
SSIM & 0.750 & 0.774 & 0.751 & 0.734 & 231 \\
Time (s) & 486 & 367 & 246 & 229 \\
\hline
\end{tabular}

TABle 8: Numerical results on Cameraman image with different mean.

\begin{tabular}{lcccc}
\hline Mean & Preserved & Not preserved & Increased & Decreased \\
\hline PSNR $(\mathrm{dB})$ & $\mathbf{2 5 . 2 1}$ & 24.34 & 19.11 & 18.22 \\
SSIM & $\mathbf{0 . 7 5 0}$ & 0.750 & 0.726 & 0.732 \\
\hline
\end{tabular}

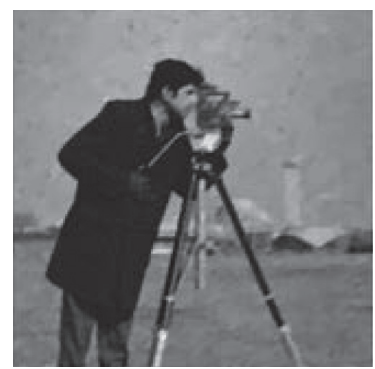

(a)

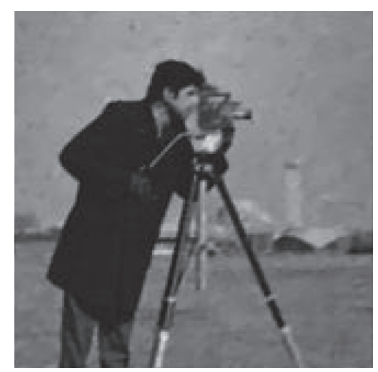

(b)

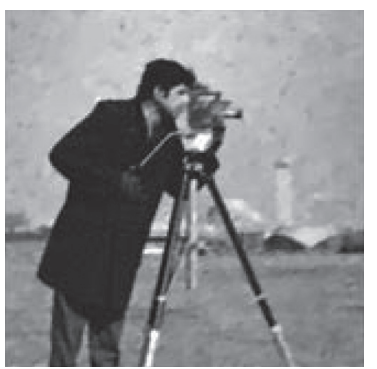

(c)

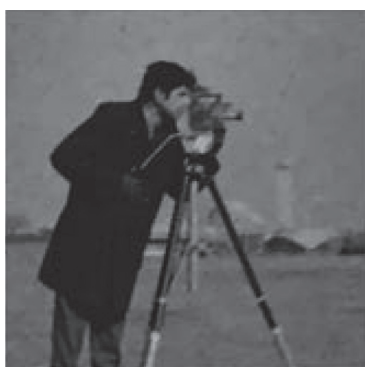

(d)

Figure 18: Results on Cameraman image with different mean: (a) preserved (25.21 dB); (b) not preserved (24.34 dB); (c) increased $(19.11 \mathrm{~dB})$; (d) decreased $(18.22 \mathrm{~dB})$.

Although there is not much difference between the recovered images obtained from preserving the mean and not preserving the mean, the PSNR value is higher when the mean is maintained as the original. These indicate that bias correction technique [36] is helpful to enhance the performance of the model (9).

\section{Conclusion}

This paper proposes a new variational model for solving the SAR image restoration problem by investigating the statistical property of multiplicative noise from Nakagami distribution. Under a mild condition, we show that the new model is strictly convex. We further analyze some mathematical properties of the model in detail. We adopt the primal-dual algorithm to solve the convex optimization problem. Two kinds of simulated experiments and real SAR images experiments are conducted. The experimental results demonstrate the excellent performance of the proposed method over some state-of-the-art methods in terms of the noise removal capability and detail preservation capability.

\section{Appendix}

The ideas for proving Propositions 1, 2, and 4 and Theorem 1 are similar to those in $[36,39]$. For readers' convenience, we present them in detail as follows.

\section{A. Proof of Proposition 1}

Proof. For any $\alpha>0$ and $\beta \geq 1$, we consider a function

$$
h(t):=2 \log t+\frac{1}{t^{2}}+\alpha(t-\beta)^{2}, \quad \forall t \in \mathbb{R}^{+},
$$

and its second order derivative is in the form of

$$
h^{\prime \prime}(t)=-2 t^{-2}+6 t^{-4}+2 \alpha \text {. }
$$

Since $t^{4} h^{\prime \prime}(t)=-2 t^{2}+6+2 \alpha t^{4}$ for $t>0$, it is not hard to show that $t^{4} h^{\prime \prime}(t)$ reaches its unique minimum $-(1 / 2 \alpha)+6$ at $t=(1 / \sqrt{2 \alpha})$. When $\alpha \geq(1 / 12)$, one can see $-(1 / 2 \alpha)+6 \geq 0$, and hence, $h^{\prime \prime}(t) \geq 0$. Moreover, since $h$ has only one minimizer, we can claim that $h$ is strictly convex if $\alpha \geq(1 / 12)$. 
For each $x \in \Omega$, we denote $u(x) / f(x)$ by $t$, and we can obtain the strict convexity of the first two terms in (6) based on the analysis above and the fact that $\log f$ is a constant. In view of the convexity of the TV regularization, we can see that $E(u)$ in (6) is strictly convex when $\alpha \geq(1 / 12)$. In addition, the feasible set $\bar{S}(\Omega)$ is convex and we can conclude that the model (6) is strictly convex when $\alpha \geq(1 / 12)$.

\section{B. Proof of Theorem 1}

Proof. We first prove that $E(u)$ in (6) is bounded from below. By the monotonic property of the function $g(s)=$ $2 \log s+\left(1 / s^{2}\right)$ on $\mathbb{R}^{+}$, we have $2 \log t+(f(x) / t)^{2} \geq$ $1+2 \log f(x)$ for any $t \in \mathbb{R}^{+} \cup\{0\}$ and $x \in \Omega$. It then follows that

$$
E(u) \geq \int_{\Omega}\left(2 \log u+\frac{f^{2}}{u^{2}}\right) \mathrm{d} x \geq \int_{\Omega}(1+2 \log f) \mathrm{d} x,
$$

which implies that $E(u)$ is bounded from below.

We next show that the solution of model (6) exists and is bounded. Let $a=\inf _{\Omega} f$ and $b=\beta \sup _{\Omega} f$. We define

$$
E_{0}(u):=\int_{\Omega}\left(2 \log u+\frac{f^{2}}{u^{2}}\right) \mathrm{d} x+\alpha \int_{\Omega}\left(\frac{u}{f}-\beta 1\right)^{2} \mathrm{~d} x .
$$

For each fixed $x \in \Omega$, we consider the following function:

$$
h(t):=2 \log t+\frac{f(x)^{2}}{t^{2}}+\alpha\left(\frac{t}{f(x)}-\beta\right)^{2}, \quad \forall t \geq 0 .
$$

It is not hard to show that $h(t)$ is decreasing on $[0, f(x))$ while increasing on $(\beta f(x),+\infty)$ and continuous on $[f(x), \beta f(x)]$. According to minimum-maximum principle, there exists at least one $\left.u^{*} \in[f(x)), \beta f(x)\right]$ that minimizes the equation $h(t)$. It then follows that $h(\min (t, M)) \leq h(t)$ if $M \geq \beta f(x)$. If we set $M=b$ and $t=u$, we immediately have

$$
E_{0}(\inf (u, b)) \leq E_{0}(u) .
$$

By $\int_{\Omega}|D \inf (u, b)| \leq \int_{\Omega}|D u|$ (see Lemma 1 in Section 4.3 of [47]), we have $E(\inf (u, b)) \leq E(u)$ [39]. Similarly, $E(\sup (u, a)) \leq E(u)$. Similar to the proof of Theorem 3.6 in [36], there exists a solution, $u^{*}$, of the model (6), satisfying $0<a \leq u^{*} \leq b$. And based on Proposition 1 and the discussion above, the uniqueness of solution immediately follows.

\section{Proof of Proposition 2}

Proof. According to theorem unique, we know that $u_{i}^{*}$ exists and it is a minimizer of $E(u)$ with $f=f_{i}$, which is defined in (6), $i=1,2$. It is clear that $E\left(\inf \left(u_{1}^{*}, u_{2}^{*}\right)\right)+$ $E\left(\sup \left(u_{1}^{*}, u_{2}^{*}\right)\right) \geq E\left(u_{1}^{*}\right)+E\left(u_{2}^{*}\right)$. Using the fact $\int_{\Omega} \mid D$ $\left(\inf \left(u_{1}^{*}, u_{2}^{*}\right)\right)\left|+\int_{\Omega}\right| D\left(\sup \left(u_{1}^{*}, u_{2}^{*}\right)\right)\left|\leq \int_{\Omega}\right| D u_{1}^{*}\left|+\int_{\Omega}\right| D u_{2}^{*} \mid$ in $[48,49]$, we get

$$
\begin{aligned}
& \int_{\Omega}\left[2 \log \left(\inf \left(u_{1}^{*}, u_{2}^{*}\right)\right)+\frac{f_{1}^{2}}{\inf \left(u_{1}^{*}, u_{2}^{*}\right)^{2}}\right. \\
& \left.+\alpha\left(\frac{\inf \left(u_{1}^{*}, u_{2}^{*}\right)}{f_{1}}-\beta 1\right)^{2}\right] \mathrm{d} x \\
& +\int_{\Omega}\left[2 \log \left(\sup \left(u_{1}^{*}, u_{2}^{*}\right)\right)+\frac{f_{2}^{2}}{\sup \left(u_{1}^{*}, u_{2}^{*}\right)^{2}}\right. \\
& \left.+\alpha\left(\frac{\sup \left(u_{1}^{*}, u_{2}^{*}\right)}{f_{2}}-\beta 1\right)^{2}\right] \mathrm{d} x \\
& \geq \int_{\Omega}\left[2 \log u_{1}^{*}+\frac{f_{1}^{2}}{\left(u_{1}^{*}\right)^{2}}+\alpha\left(\frac{u_{1}^{*}}{f_{1}}-\beta 1\right)^{2}\right] \mathrm{d} x \\
& \quad+\int_{\Omega}\left[2 \log u_{2}^{*}+\frac{f_{2}^{2}}{\left(u_{2}^{*}\right)^{2}}+\alpha\left(\frac{u_{2}^{*}}{f_{2}}-\beta 1\right)^{2}\right] \mathrm{d} x .
\end{aligned}
$$

Note that $\Omega=\left\{u_{1}^{*}>u_{2}^{*}\right\} \cup\left\{u_{1}^{*} \leq u_{2}^{*}\right\}$. One can see

$$
\begin{aligned}
& \int_{\left\{u_{1}^{*}>u_{2}^{*}\right\}}\left(f_{1}-f_{2}\right)\left(u_{1}^{*}-u_{2}^{*}\right)\left[\left(f_{1}+f_{2}\right)\left(u_{1}^{*}+u_{2}^{*}\right)\right. \\
& \left.\cdot \frac{1}{u_{1}^{* 2} u_{2}^{* 2}}+\left(f_{1}+f_{2}\right)\left(u_{1}^{*}+u_{2}^{*}\right) \frac{\alpha}{f_{1}^{2} f_{2}^{2}}-2 \alpha \beta \frac{1}{f_{1} f_{2}}\right] \geq 0 .
\end{aligned}
$$

Define $h(x):=\left(f_{1}+f_{2}\right)\left(u_{1}^{*}+u_{2}^{*}\right)\left(1 / u_{1}^{* 2} u_{2}^{* 2}\right)+\left(f_{1}+\right.$ $\left.f_{2}\right)\left(u_{1}^{*}+u_{2}^{*}\right)\left(\alpha / f_{1}^{2} f_{1}^{2}\right)-2 \alpha \beta\left(1 / f_{1} f_{2}\right)$. Based on Theorem 1 , we have $0<a_{1} \leq u_{1}^{*} \leq b_{1}$ and $0<a_{2} \leq u_{2}^{*} \leq b_{2}$, which yields

$$
\begin{gathered}
h(x) \geq\left(a_{1}+a_{2}\right)\left(a_{1}+a_{2}\right) \frac{1}{b_{1}^{2} b_{2}^{2}}+\left(a_{1}+a_{2}\right)\left(a_{1}+a_{2}\right) \frac{\alpha}{b_{1}^{2} b_{2}^{2}} \\
-\frac{2 \alpha \beta}{a_{1} a_{2}} \geq \frac{2 a_{1} a_{2}}{b_{1}^{2} b_{2}^{2}}+\frac{2 \alpha a_{1} a_{2}}{b_{1}^{2} b_{2}^{2}}-\frac{2 \alpha \beta}{a_{1} a_{2}} .
\end{gathered}
$$

Hence, one can see that

$$
\frac{2 a_{1} a_{2}}{b_{1}^{2} b_{2}^{2}}+\frac{2 \alpha a_{1} a_{2}}{b_{1}^{2} b_{2}^{2}}-\frac{2 \alpha \beta}{a_{1} a_{2}}>0,
$$

given $(\alpha \beta /(1+\alpha))<\left(a_{1}^{2} a_{2}^{2} / b_{1}^{2} b_{2}^{2}\right)$. This together with the fact that $f_{1}<f_{2}$ implies that $\left\{u_{1}^{*}>u_{2}^{*}\right\}$ has a zero Lebesgue measure, i.e., $u_{1}^{*} \leq u_{2}^{*}$ a.e. in $\Omega$.

\section{Proof of Proposition 4}

Proof. Let $C$ be the minimum of (9), which is independent of $\varepsilon$. Set $w=\left(K u^{*} / f\right)$. We then have

$$
\int_{\Omega}\left(2 \log w+\frac{1}{w^{2}}\right) \mathrm{d} x \leq C-2 \int_{\Omega} \log f \mathrm{~d} x .
$$


Since $h(t)=2 \log t+\left(1 / t^{2}\right)$ is decreasing on $(0,1)$ and increasing on $(1,+\infty)$, it is obvious that $2 \log t+\left(1 / t^{2}\right) \geq 1$ for $t>0$, which immediately implies that

$$
|\Omega| \leq \int_{\Omega}\left(2 \log w+\frac{1}{w^{2}}\right) \mathrm{d} x .
$$

When $t \leq \varepsilon<1$, we have $2 \log t+\left(1 / t^{2}\right) \geq 2 \log \varepsilon+\left(1 / \varepsilon^{2}\right)$, which together with (D.1) and (D.2) yields

$$
\begin{gathered}
|\{x \in \Omega: w(x) \leq \varepsilon\}|\left(2 \log \varepsilon+\frac{1}{\varepsilon^{2}}\right) \leq \int_{\{x \in \Omega: \omega(x) \leq \varepsilon\}} \\
\cdot\left(2 \log \omega+\frac{1}{\omega^{2}}\right) \mathrm{d} x \leq C-2 \int_{\Omega} \log f \mathrm{~d} x .
\end{gathered}
$$
holds.

Since $w=\left(K u^{*} / f\right)$ and $\inf _{\Omega} f>0$, the conclusion

\section{Data Availability}

All data generated or analysed during this study are included within the article.

\section{Conflicts of Interest}

The authors declare that there are no conflicts of interest.

\section{Acknowledgments}

The authors would like to thank Dr. Zhaosong $\mathrm{Lu}$ for valuable comments on an earlier draft of the manuscript. This work was supported by the Natural Science Foundation of Guangdong Province of China under grant 2020B1515310008 and the Project of Educational Commission of Guangdong Province of China under grant 2019KZDZX1007.

\section{References}

[1] A. Beck and M. Teboulle, "Fast gradient-based algorithms for constrained total variation image denoising and deblurring problems," IEEE Transactions on Image Processing, vol. 18, no. 11, pp. 2419-2434, 2009.

[2] A. A. Bini and M. S. Bhat, "A nonlinear level set model for image deblurring and denoising," The Visual Computer, vol. 30 , no. 3, pp. 311-325, 2014.

[3] J.-F. Cai, H. Ji, Z. Shen, and G.-B. Ye, "Data-driven tight frame construction and image denoising," Applied and Computational Harmonic Analysis, vol. 37, no. 1, pp. 89-105, 2014.

[4] R. H. Chan and J. Ma, "A multiplicative iterative algorithm for box-constrained penalized likelihood image restoration," IEEE Transactions on Image Processing, vol. 21, no. 7, pp. 3168-3181, 2012.

[5] W. Gao, G. Song, and Y. Zhang, "PCM-TV-TFV: a novel two stage framework for image reconstruction from Fourier data," SIAM Journal on Imaging Sciences, vol. 10, no. 4, pp. 22502274, 2017.

[6] J. Lu, Z. Ye, and Y. Zou, "Huber fractal image coding based on a fitting plane," IEEE Transactions on Image Processing: A Publication of the IEEE Signal Processing Society, vol. 22, no. 1, pp. 134-145, 2013.

[7] J. Lu, K. Qiao, X. Li, Z. Lu, and Y. Zou, " $l_{0}$-minimization methods for image restoration problems based on wavelet frames," Inverse Problems, vol. 35, no. 6, Article ID 064001, 2019.

[8] S. Luo and T. Zhou, "Superiorization of EM algorithm and its application in single-photon emission computed tomography (SPECT)," Inverse Problems and Imaging, vol. 8, no. 1, pp. 223-246, 2014.

[9] Y. Wang, J. Guo, W. Chen, and W. Zhang, "Image denoising using modified Perona-Malik model based on directional Laplacian," Signal Processing, vol. 93, no. 9, pp. 2548-2558, 2013.

[10] F. Wang, X.-L. Zhao, and M. K. Ng, "Multiplicative noise and blur removal by framelet decomposition and $l_{1}$-based L-curve method," IEEE Transactions on Image Processing, vol. 25, no. 9, pp. 4222-4232, 2016.

[11] Y. Chen, W. Feng, R. Ranftl, H. Qiao, and T. Pock, “A higherorder MRF based variational model for multiplicative noise reduction," IEEE Signal Processing Letters, vol. 21, no. 11, pp. 1370-1374, 2014.

[12] J. P. Fitch, "Synthetic aperture radar," in Signal Processing and Digital Filtering, Springer, Berlin, Germany, 1988.

[13] J. W. Goodman, "Statistical properties of laser speckle patterns," Laser Speckle and Related Phenomena, vol. 9, pp. 9-75, Springer, Berlin, Germany, 1975.

[14] A. Marino, "Synthetic aperture radar," A New Target Detector Based on Geometrical Perturbation Filters for Polarimetric Synthetic Aperture Radar (POL-SAR), pp. 9-27, Springer, Berlin, Germany, 2012.

[15] D. C. Munson and R. L. Visentin, "A signal processing view of strip-mapping synthetic aperture radar," IEEE Transactions on Acoustics, Speech, and Signal Processing, vol. 37, no. 12, pp. 2131-2147, 1989.

[16] Y.-M. Huang, D.-Y. Lu, and T. Zeng, "Two-step approach for the restoration of images corrupted by multiplicative noise," SIAM Journal on Scientific Computing, vol. 35, no. 6, pp. A2856-A2873, 2013.

[17] Y. Huang, M. Ng, and T. Zeng, "The convex relaxation method on deconvolution model with multiplicative noise," Communications in Computational Physics, vol. 13, no. 4, pp. 1066-1092, 2013.

[18] Z. Li, Y. Lou, and T. Zeng, "Variational multiplicative noise removal by DC programming," Journal of Scientific Computing, vol. 68, no. 3, pp. 1200-1216, 2016.

[19] J. Lu, L. Shen, C. Xu, and Y. Xu, "Multiplicative noise removal with a sparsity-aware optimization model," Inverse Problems \& Imaging, vol. 11, no. 6, pp. 949-974, 2017.

[20] J.-S. Lee, "Digital image enhancement and noise filtering by use of local statistics," IEEE Transactions on Pattern Analysis and Machine Intelligence, vol. 2, no. 2, pp. 165-168, 1980.

[21] G. T. Li, C. L. Wang, P. P. Huang, and W. D. Yu, "SAR image despeckling using a space-domain filter with alterable window," IEEE Geoscience and Remote Sensing Letters, vol. 10, no. 2, pp. 263-267, 2013.

[22] L. Ji, Y. Xu, and Y. Zhang, "Simulation of linear and nonlinear advection-diffusion-reaction problems by a novel localized scheme," Applied Mathematics Letters, vol. 99, Article ID 106005, 2020.

[23] J. Yang, X. Zhao, T. Ma, Y. Chen, T. Huang, and M. Ding, "Remote sensing images destriping using unidirectional hybrid total variation and nonconvex low-rank regularization," Journal of Computational and Applied Mathematics, vol. 363, pp. 124-144, 2020.

[24] F. Argenti, T. Bianchi, A. Lapini, and L. Alparone, "Fast MAP despeckling based on Laplacian-Gaussian modeling of wavelet 
coefficients," IEEE Geoscience and Remote Sensing Letters, vol. 9, no. 1, pp. 12-17, 2012.

[25] T. Bianchi, F. Argenti, and L. Alparone, "Segmentation-based MAP despeckling of SAR images in the undecimated wavelet domain," IEEE Transactions on Geoscience and Remote Sensing, vol. 46, no. 9, pp. 2728-2742, 2008.

[26] D. Cozzolino, S. Parrilli, G. Scarpa, G. Poggi, and L. Verdoliva, "Fast adaptive nonlocal SAR despeckling," IEEE Geoscience and Remote Sensing Letters, vol. 11, no. 2, pp. 524-528, 2014.

[27] C.-A. Deledalle, L. Denis, and F. Tupin, "Iterative weighted maximum likelihood denoising with probabilistic patchbased weights," IEEE Transactions on Image Processing, vol. 18, no. 12, pp. 2661-2672, 2009.

[28] S. Parrilli, M. Poderico, C. V. Angelino, and L. Verdoliva, "A nonlocal SAR image denoising algorithm based on LLMMSE wavelet shrinkage," IEEE Transactions on Geoscience and Remote Sensing, vol. 50, no. 2, pp. 606-616, 2012.

[29] X. Zhao, F. Wang, and K. N. Michael, "A new convex optimization model for multiplicative noise and blur removal," SIAM Journal on Imaging Sciences, vol. 7, pp. 456-475, 2014.

[30] Y.-M. Huang, M. K. Ng, and Y.-W. Wen, "A new total variation method for multiplicative noise removal," SIAM Journal on Imaging Sciences, vol. 2, no. 1, pp. 20-40, 2009.

[31] Y. Huang, L. Moisan, M. K. Ng, and T. Zeng, "Multiplicative noise removal via a learned dictionary," IEEE Transactions on Image Processing: A Publication of the IEEE Signal Processing Society, vol. 21, no. 11, pp. 4534-4543, 2012.

[32] J. Lu, L. Shen, C. Xu, and Y. Xu, "Multiplicative noise removal in imaging: an exp-model and its fixed-point proximity algorithm," Applied and Computational Harmonic Analysis, vol. 41, no. 2, pp. 518-539, 2016.

[33] J. Black and S. Osher, "A nonlinear inverse scale space method for a convex multiplicative noise model," SIAM Journal on Imaging Sciences, vol. 1, no. 3, pp. 294-321, 2008.

[34] S. Yun and H. Woo, "A new multiplicative denoising variational model based on $m$-th root transformation," IEEE Transactions on Image Processing, vol. 21, pp. 2523-2533, 2012.

[35] M. Kang, S. Yun, and H. Woo, "Two-level convex relaxed variational model for multiplicative denoising," SIAM Journal on Imaging Sciences, vol. 6, no. 3, pp. 875-903, 2013.

[36] Y. Dong and T. Zeng, "A convex variational model for restoring blurred images with multiplicative noise," SIAM Journal on Imaging Sciences, vol. 6, no. 3, pp. 1598-1625, 2013.

[37] N. Tabassum, A. Vaccari, and S. Acton, "Speckle removal and change preservation by distance-driven anisotropic diffusion of synthetic aperture radar temporal stacks," Digital Signal Processing, vol. 74, pp. 43-55, 2018.

[38] S. Roth and M. J. Black, "Fields of experts," International Journal of Computer Vision, vol. 82, no. 2, pp. 205-229, 2009.

[39] G. Aubert and J.-F. Aujol, "A variational approach to removing multiplicative noise," SIAM Journal on Applied Mathematics, vol. 68, no. 4, pp. 925-946, 2008.

[40] N. Komodakis and J.-C. Pesquet, "Playing with duality: an overview of recent primal dual approaches for solving largescale optimization problems," IEEE Signal Processing Magazine, vol. 32, no. 6, pp. 31-54, 2015.

[41] T. Goldstein and S. Osher, "The split Bregman method for L1regularized problems," SIAM Journal on Imaging Sciences, vol. 2, no. 2, pp. 323-343, 2009.

[42] S. Boyd, N. Parikh, E. Chu, B. Peleato, and J. Eckstein, "Distributed optimization and statistical learning via the alternating direction method of multipliers," Foundations and Trends in Machine Learning, vol. 3, no. 1, pp. 1-122, 2011.
[43] C. A. Micchelli, L. Shen, and Y. Xu, "Proximity algorithms for image models: denoising," Inverse Problems, vol. 27, no. 4, Article ID 045009, 2011.

[44] A. Chambolle and T. Pock, "A first-order primal-dual algorithm for convex problems with applications to imaging," Journal of Mathematical Imaging and Vision, vol. 40, no. 1, pp. 120-145, 2011.

[45] Y. Yu and S. T. Acton, "Speckle reducing anisotropic diffusion," IEEE Transactions on Image Processing: A Publication of the IEEE Signal Processing Society, vol. 11, no. 11, pp. 12601270, 2002.

[46] Z. Wang, A. C. Bovik, H. R. Sheikh, and E. P. Simoncelli, "Image quality assessment: from error visibility to structural similarity," IEEE Transactions on Image Processing, vol. 13, no. 4, pp. 600-612, 2004.

[47] P. Kornprobst, R. Deriche, and G. Aubert, "Image sequence analysis via partial differential equations," Journal of Mathematical Imaging and Vision, vol. 11, no. 1, pp. 5-26, 1999.

[48] A. Chambolle, "An algorithm for total variation minimization and applications," Journal of Mathematical Imaging and Vision, vol. 20, no. 1, pp. 89-97, 2014.

[49] E. Giusti, "Minimal surfaces and functions of bounded variation," Monographs in Mathematics, Vol. 80, Springer, Berlin, Germany, 1984. 